\title{
DECENTRALISED AUTONOMOUS ORGANISATIONS AND THE CORPORATE FORM
}

\author{
Nathan Tse*
}

\begin{abstract}
It has been suggested that the development of decentralised autonomous organisations (DAOs) will lead to a paradigm shift in the way we perceive businesses. DAOs ostensibly eliminate agency costs due to the absence of a board of directors, automated governance mechanisms and transparency provided by the blockchain upon which the DAO is launched. This article undertakes a comparative analysis between DAOs and corporations and questions whether DAOs really do improve the corporate form. Using a corporate governance and legal realist lens, this article suggests that a number of the purported benefits of DAOs are overly simplified. Moreover, there are several practical and legal obstacles that technological advancements and improved engineering must overcome before DAOs become a viable, mainstream organisational structure. Balancing the inevitable improvement in technology against these significant obstacles, this article predicts an incremental integration of DAOs into society through a hybrid approach, involving interim legal solutions and varying degrees of automation and decentralisation.
\end{abstract}

\section{INTRODUCTION}

The 16th and 17th centuries witnessed a fundamental change in the way businesses operate. ${ }^{1}$ Expansive trade operations demanded long-term investment which could not be ascertained through typical partnership structures. ${ }^{2}$ The economic need for the secure investment of capital, without risk of private or public expropriation, was a major driver for the establishment of the corporate form. ${ }^{3}$ The public listing of the Dutch East India Company in 1602 was influential in the way businesses ran,

\footnotetext{
* Law Clerk, Russell McVeagh. Submitted for the LLB (Honours) Degree, Faculty of Law, Victoria University of Wellington, 2019. Recipient of the Legal Research Foundation Unpublished Undergraduate Student Paper Award.

1 Giuseppe Dari-Mattiacci and others "The Emergence of the Corporate Form" (2017) 33 JLEO 193 at 193.

2 At 193

3 At 193
} 
forming the basis of modern corporate governance. ${ }^{4}$ The development of a corporation's five core structural characteristics-legal personality, limited liability, transferable shares, centralised management under a board structure and shared ownership by capital contributors - have made the modern corporation "uniquely attractive for organizing productive activity". 5

However, with corporations now being some of the strongest entities in the world, corporate scandals, such as the collapse of Enron, WorldCom and Parmalat, have shown that the corporate form is not infallible. ${ }^{6}$ Corporate governance literature highlights the need for incentive mechanisms and structural safeguards to overcome divergent interests between shareholders, managers and external stakeholders. Despite robust internal processes outlined in company constitutions and the codification of industry best practices in legislation, ${ }^{7}$ empirical analyses suggest that modern corporations remain susceptible to failure. ${ }^{8}$

Four hundred years after the Dutch East India Company's public listing, decentralised autonomous organisations (DAOs) purportedly represent a brand new "innovation in the design of organizations". 9 Built upon a foundation of blockchain technology, DAOs (or DACs, decentralised autonomous corporations) ostensibly circumvent traditional principal-agent relationships, transforming the way we perceive governance.

Proponents and enthusiasts say that DAOs will eventually replace many of the world's corporations. Although this may seem farfetched for most traditionalists, DAOs continue to expand their potential capabilities in conjunction with technological advancements. It is these unknown future capabilities of DAOs which lead so-called "crypto-anarchists" to proclaim that DAOs represent a shift towards a decentralised autonomous society "in which humans are 'freed' from centralized institutions of power and control". ${ }^{10}$

4 At 193

5 John Armour, Henry Hansmann and Reinier Kraakman " What Is Corporate Law?" in Reinier Kraakman and others The Anatomy of Corporate Law: A Comparative and Functional Approach (2nd ed, Oxford University Press, New York, 2009) 1 at 6.

6 Ana Paula Paulino da Costa "Corporate Governance and Fraud: Evolution and Considerations" in Okechukwu Lawrence Emeagwali (ed) Corporate Governance and Strategic Decision Making (Girne American University, Girne (Cyprus), 2017) 1 at 1.

7 See for example New Zealand's Companies Act 1993 which includes a comprehensive list of default rules for companies to adopt, based on market practice.

8 Da Costa, above n 6, at 1.

9 Usman W Chohan The Decentralized Autonomous Organization and Governance Issues (University of New South Wales, Discussion Paper, December 2017) at 1.

10 JZ Garrod "The Real World of the Decentralized Autonomous Society" (2016) 14 tripleC 62 at 62. 
Despite the somewhat seductive features of this decentralised utopia, this article questions whether a comprehensive uptake of DAOs in our everyday life is truly feasible. For enthusiasts, the fact that DAOs are underpinned by a number of established theories, from game theory to information technology governance, is a source of legitimacy. ${ }^{11}$ However, by detaching the theoretical benefits from the practical obstacles of DAO proliferation, enthusiasts undermine their arguments for a decentralised future.

Firstly, this article undertakes a comparative analysis between the corporate form and these new decentralised organisational structures, using both a corporate governance and legal realist lens. In doing so, it outlines a number of complexities and costs pertaining to an organisation's decentralised governance structure. The following section of the article delineates three key obstacles faced by DAOs: legal indeterminacy, a tendency towards centralisation and incumbent institutions. Finally, wary of the risk tied to dismissing technological innovations, this article predicts that DAOs will not permeate society in a way envisaged by most crypto-anarchists, but will instead incrementally integrate into society via a hybrid approach.

\section{DO DECENTRALISED AUTONOMOUS ORGANISATIONS (DAOS) IMPROVE THE CORPORATE FORM?}

The following Part canvasses some of the purported benefits of DAOs, when compared to conventional company structures. Sub-part A provides a workable definition of a DAO and explains how they operate with reference to blockchain technology, smart contracts and their modus operandi. Sub-part B analyses exactly how DAOs transform corporate governance, with reference to specific agency costs so as to provide context regarding the issues DAOs ostensibly overcome. Throughout the analysis, attention is drawn to the complexities of DAO governance. There is undoubtedly merit in the greater use of decentralised systems. However apparent problems with governance, technology and legalistic hurdles hinder the expansion of DAOs in both the business world and society at large.

\section{A Explaining DAOs}

Before outlining how DAOs purportedly overcome conventional corporate issues, it is necessary to get a comprehensive understanding of what we mean by a DAO and how DAOs operate.

11 Roman Beck, Christoph Müller-Bloch and John Leslie King "Governance in the Blockchain Economy: A Framework and Research Agenda" (2018) 19 JAIS 1020 at 1021. 


\section{Defining DAOs}

Like many contemporary developments, decentralised autonomous organisations do not have one accepted definition. Jack du Rose, co-founder of Colony, ${ }^{12}$ defines a DAO as: ${ }^{13}$

... a type of decentralised application which incentivises its users to engage in activity which furthers its agreed business objectives by enabling them to work together without requiring them to trust one another.

Du Rose's definition encompasses a range of decentralised applications, from the Ethereum based venture capitalist fund, The DAO, to the world's first cryptocurrency, Bitcoin. This definition hints towards the optimisation proposal process employed by a typical DAO. ${ }^{14}$ This process involves users putting forward proposals that will optimise the value of the DAO. A slightly more precise definition labels a DAO "an organization that is run through rules encoded as computer programs called 'smart contracts'". ${ }^{15}$ This is not mutually exclusive with du Rose's definition, but rather emphasises the role played by smart contracts, deployed on a blockchain, in the operation of a DAO.

In contrast to du Rose, Primavera De Filippi and Aaron Wright distinguish between forms of decentralised organisations where, on the one hand, the ultimate decision-making power resides in humans, and DAOs which, on the other hand, are controlled "entirely by code". ${ }^{16}$ Both forms of decentralised organisation resemble a fundamental shift in the way organisations operate, and therefore both will be considered within this article. Rather than classify them as distinct classes of entity, it is preferable to perceive both as DAOs which fall on varying levels of an autonomous spectrum.

\section{A brief summary of blockchain}

Put simply, a blockchain is a decentralised database or ledger that is distributed between nodes in a peer to peer network. ${ }^{17}$ Each user or "node" in the network can access a replicate of the ledger, and

12 Colony (2019) <https://colony.io>.

13 Jack du Rose "Clearmatics, EtherCasts \& Colony" (speech to the London Ethereum Monthly Meetup, London, 4 May 2016).

14 Wulf A Kaal "Blockchain Solutions for Agency Problems in Corporate Governance" in Kashi R Balachandran (ed) Economic Information to Facilitate Decision Making (World Scientific Publishers, Singapore, 2019) 1 at 19 .

15 Chohan, above $\mathrm{n} 9$, at 1.

16 Primavera De Filippi and Aaron Wright Blockchain and the Law: The Rule of Code (Harvard University Press, Cambridge (Mass), 2018) at 148.

17 Alex Norta "Creation of Smart-Contracting Collaborations for Decentralized Autonomous Organizations" in Raimundas Matulevičius and Marlon Dumas (eds) Perspectives in Business Informatics Research (14th International Conference, BIR 2015, Tartu, Estonia, 26-28 August 2015) 2015) 3 at 3. 
community validation is used to keep the ledger content synchronised. ${ }^{18}$ Blockchains thereby circumvent the need for a trusted third party to validate transactions, as the network instead validates transactions by consensus. ${ }^{19}$ This community validation process (or consensus protocol) is a set of formalised, pre-defined governance rules stored on the blockchain's consensus layer. ${ }^{20}$

Anyone with an Internet connection can view information stored on a blockchain by downloading freely available open source software. ${ }^{21}$ Consequently, as any node in the network can update the ledger, public blockchains are not controlled by any one centralised party. Built on a peer to peer network, blockchains encourage disintermediation, ${ }^{22}$ making them ideal for circumventing central bodies, whether that is a financial intermediary or a board of directors. ${ }^{23}$

\section{Smart contracts}

Smart contracts are computerised transaction protocols which, in theory, execute contractual terms. ${ }^{24}$ Essentially, smart contracts operate as computer programmes which are deployed on a blockchain, ${ }^{25}$ so that they are non-repudiable and verifiable. ${ }^{26}$

Before proceeding, it would be remiss not to acknowledge the literature around the limitations of so-called "computable contracts", ${ }^{27}$ such as smart contracts, outlined by a number of academics. ${ }^{28}$ Simply put, the complexity of contractual terms in computable contracts is limited due to a natural language processing problem, issues of interpreting abstract or subjective concepts and concerns around areas of contractual uncertainty. ${ }^{29}$ Conventional legal contracts are written in legalistic

18 Tomaso Aste, Paolo Tasca and Tiziana Di Matteo "Blockchain Technologies: The Foreseeable Impact on Society and Industry" (2017) 50(9) Computer 18 at 18.

19 Voshmgir Shermin "Disrupting governance with blockchains and smart contracts" (2017) 26 Strategic Change 499 at 499.

20 At 499

21 De Filippi and Wright, above n 16, at 34.

22 At 34

23 Marcella Atzori "Blockchain Technology and Decentralized Governance: Is the State Still Necessary?" (PhD Thesis, University of Nicosia, Cyprus, 2015) at 15.

24 Norta, above n 17 , at 3.

25 Soichiro Takagi "Organizational Impact of Blockchain through Decentralized Autonomous Organizations" (2017) 12 IJEPS 22 at 25.

26 Norta, above n 17 , at 3.

27 Harry Surden "Computable Contracts" (2012) 46 UC Davis L Rev 629 at 642.

28 James Grimmelmann "All Smart Contracts are Ambiguous" (2019) 2 Journal of Law \& Innovation 1.

29 Surden, above n 27, at 643. 
language, and may include ambiguous terms such as "reasonable" or "best efforts" which create disputes over interpretation. Surden argues that these limitations can, in certain circumstances, be circumvented by data-oriented contracts, which are contracts "in which the parties have expressed one or more terms or conditions of their agreement in a manner designed to be processable by a computer system". ${ }^{30}$ Likewise one can use computer semantics to translate a legal contract into computer processable rules ${ }^{31}$ or provide a computer with a database that it can automatically access to check compliance or performance. ${ }^{32}$ It is logical, therefore, that smart contracts take the form of dataoriented contracts, which can be processed automatically on the blockchain using the underlying blockchain's programming language (for example: Solidity on the Ethereum blockchain).

Ensuring smart contracts, which mimic legal agreements, are properly translated into dataoriented contracts may not solve all issues with complexity. Surden argues that data-oriented contracts are only suitable in standardised scenarios with factual certainty, and for agreements which are able to be decomposed into computer processable terms. ${ }^{33}$ However, since Surden's seminal piece on computable contracts, new technological developments have increased the level of contractual complexity comprehendible by computers. ${ }^{34}$ Furthermore, the future employment of machine learning technology and artificial intelligence in smart contracts exponentially increases the capability to mirror traditional legal agreements.

In acknowledgement that the complexity of smart contracts is limited, but ever increasing, it would be naïve to say that they will not have far-reaching implications in the future. Nevertheless, a smart contract's most important feature is that it is not only defined, but also executed by its underlying code. ${ }^{35}$ Once the contractual parameters (codified in the smart contract) are fulfilled, the contract is automatically enforced "without discretion", meaning that, all things equal, promisees do not face the risk of losing their end of the bargain. ${ }^{36}$

30 At 639 .

31 At 665

32 At 671

33 At 682 .

34 See for example Sudhir Agarwal, Kevin Xu and John Moghtader "Toward Machine-Understandable Contracts" (paper presented to the 22nd European Conference on Artificial Intelligence, The Hague, The Netherlands, 30 August 2016).

35 Melanie Swan Blockchain: Blueprint for a New Economy (O'Reilly, California, 2015) at ch 2, as quoted in Takagi, above n 25, at 25 .

36 At 25. 
Broadly speaking, a smart contract on a public blockchain will go through four distinct stages: creation, acceptance, execution and result confirmation. ${ }^{37}$ Firstly, users will create the contract using digital signatures to guarantee its authenticity. ${ }^{38}$ Secondly, users who receive the smart contract will check its validity and mine to include it in a new block, where it will be broadcasted to the blockchain at large. ${ }^{39}$ Thirdly, users will execute the smart contract, according to its instructions. Once the result is obtained, users will need to mine to include the result in a new block, which is then broadcasted to the blockchain. ${ }^{40}$ Finally, users who receive a block containing the result will verify its correctness (often by re-computing the smart contract and comparing the result with that which is received) and determine whether to accept it or not. ${ }^{41}$

The automation of smart contracts involves the deployment of algorithms that can "self-execute, self-enforce, self-verify, and self-constrain the performance of the contracts". ${ }^{42}$ A smart contract can be coded such that, when it is executed, it triggers another smart contract, enabling the execution of a chain of smart contracts. It is the automatic self-executing feature of smart contracts which enables the creation of decentralised organisations. ${ }^{43}$

\section{How DAOs operate}

By engineering more complex smart contracts, organisations can be established, where the rules of governance are defined in code on a blockchain. ${ }^{44}$ With the rules of governance being transparent, and distributed to all nodes in the network, DAOs do not have any single "owner" who can directly force them to act in a particular way. ${ }^{45}$ DAOs therefore enable a form of non-hierarchical governance, where decision-making power is spread across the network's nodes rather than deferred to a centralised body. ${ }^{46}$

37 Nour Diallo and others "eGov-DAO: A Better Government using Blockchain based Decentralized Autonomous Organization" (paper presented to the Fifth International Conference on eDemocracy and eGovernment, Quito, Ecuador, 4-6 April 2018) at 167.

38 At 167 .

39 At 167

40 At 167 .

41 At 167

42 Aste, Tasca and Di Matteo, above n 18, at 19 (italics omitted).

43 At 19.

44 Diallo and others, above n 37, at 167.

45 De Filippi and Wright, above n 16, at 149.

46 Aste, Tasca and Di Matteo, above n 18, at 23. 
A DAO's duty is to abide by its specific programmatic set of rules ${ }^{47}$ so in theory, a DAO can be set for any purpose or objective. ${ }^{48}$ A DAO's architecture may determine whether it is capable of carrying out a "specific and deterministic task", or something more sophisticated where people or machines interact to achieve a specific purpose ${ }^{49}$ Ultimately, the way a DAO operates depends on its degree of automation.

At the lower end of the autonomous spectrum, a DAO may act as a platform, where members interact according to a self-enforcing, open source protocol. ${ }^{50}$ Those interested in furthering the objective of the DAO (or at least interested in optimising the value of the DAO) purchase the DAO's tokens. ${ }^{51}$ These tokens give voting rights to members, who can then vote on proposals put forward by other DAO members (for instance, to undertake a new project). ${ }^{52}$ It is in the interests of all DAO token holders that only beneficial proposals that will optimise the value of the DAO are approved. ${ }^{53}$ If the proposal is approved, it will be recorded in the blockchain. Remuneration for fulfilling the proposal will typically be codified in a smart contract, such that compensation will only be rewarded once proposers deliver on their promise. ${ }^{54}$

DAOs with this architecture have the potential to retain a strong human element, as DAO members (who can be humans or machines) still vote on decisions, and put forward their own proposals. Despite this human element, with governance rules codified in smart contracts, relevant governance decisions are still automatically executed without manual intervention, circumventing the need for a central decision-making entity. ${ }^{55}$

At the far end of the autonomous spectrum are DAOs which incorporate artificial intelligence to run entirely autonomously on a blockchain. Activities of this type of DAO are fully determined by a blockchain's protocol and the DAO's smart contract code. ${ }^{56}$ As a DAO can use digital tokens to trigger smart contracts independently, ultimately, with a sufficient number of digital tokens to pay a

47 BlockChannel "What Is A 'DAO'? How Do They Benefit Consumers?" (22 March 2016) Medium $<$ https://medium.com>.

48 Diallo and others, above $\mathrm{n} 37$, at 167

49 De Filippi and Wright, above n 16, at 148.

50 See Shermin Voshmgir Token Economy: How Blockchains and Smart Contracts Revolutionize the Economy (BlockchainHub, Berlin, 2019).

51 Kaal, above n 14, at 19.

52 At 20

53 At 19.

54 At 20

55 Diallo and others, above n 37, at 167.

56 De Filippi and Wright, above n 16, at 148. 
blockchain network for the resources it needs, a DAO can operate indefinitely without human control. ${ }^{57}$ Humans may still contribute funds to the DAO in return for digital tokens (and a share in the DAO's profits), or interact with DAOs by paying for its service. ${ }^{58}$

These DAOs can be constructed in two distinct ways. The most direct way is to embed decisionmaking capabilities in the DAO's smart contract code. ${ }^{59}$ The underlying algorithm may interact with environmental inputs (including the needs and desires of people), but no person can exert direct influence over its operations. ${ }^{60}$ In this way, the DAO's underlying code comprises all requirements needed to complete a task. ${ }^{61}$

A DAO can also be constructed by the coordinated aggregation of multiple smart contracts, creating "a DAO whose capabilities are much greater than the sum of its parts". 62 De Filippi and Wright argue that these more sophisticated DAOs benefit from the collective intelligence of a number of independent smart contracts which may purposefully or inadvertently contribute to achieving a common goal. ${ }^{63}$

\section{B Transforming Governance with DAOs}

The corporate form has facilitated the advancement of commerce in a multitude of ways. Principles of agency allow for the efficient delegation of decision-making. ${ }^{64}$ Limited liability shields a shareholder's personal assets from company creditors, incentivising positive risk-taking. ${ }^{65}$ Similarly, entity shielding protects company assets from a shareholder's personal creditors, enabling the facilitation of credit. ${ }^{66}$ The "locking in" of capital ensures that companies have a right to retain their capital and can more securely engage in long-term investment. ${ }^{67}$

However, the characterisation of competing interests between stakeholders, shareholders and managers as principal-agent relationships has uncovered significant costs and inefficiencies inherent
57 At 148
58 At 149
59 At 149
60 At 149 .
61 Diallo and others, above $\mathrm{n} 37$, at 167.
62 De Filippi and Wright, above n 16, at 149.
63 At 150
64 Dari-Mattiacci and others, above $\mathrm{n} 1$, at 1.
65 At 1.
66 At 1.
67 At 1. 
in the corporate form. ${ }^{68}$ This is most noticeably characterised by the principal-agent relationship pertaining to the "separation of ownership and control". ${ }^{69}$ Shareholders (the principals) who delegate decision-making responsibility to managers (the agents) face the risk of the managers taking advantage of asymmetrical information, acting opportunistically, and pursing their own personal interests rather than promoting the welfare of the shareholders. ${ }^{70}$ Similar agency problems involve the divergent interests of the controlling shareholders and non-controlling shareholders of the firm, ${ }^{71}$ and conflicts between a firm itself and external stakeholders, such as creditors, employees, customers and the environment. ${ }^{72}$

In light of these enduring issues, DAO proponents argue that blockchain technology could reduce the need for businesses to organise as companies altogether. ${ }^{73}$ Overcoming agency costs, such as those described above, are the predominant justification for the use of DAOs. However, those more sceptical might argue that the governance problems DAOs ostensibly diminish are merely replaced by different ones. This section of the article explains some of the purported improvements that decentralised systems bring to the governance of organisations, with explicit referral to conventional agency costs. In doing so, it critiques the simplistic nature that these improvements are often canvassed and raises some complexities which ought to be considered.

\section{Company boards, hierarchies and decentralised decision-making}

The decision-making powers of a corporation are typically vested in the board of directors, subject to any constitutional constraints and special resolutions made by shareholders. ${ }^{74}$ This means that decisions pertaining to issues such as a company's strategic direction or employee welfare are made by one internal, central body. ${ }^{75}$ The board therefore has significant power over the future prospects of the company. As mentioned below, the separation of ownership and control-embodied by a

68 Michael C Jensen and William H Meckling "Theory of the Firm: Managerial Behavior, Agency Costs and Ownership Structure" (1976) 3 JFE 305 at 308.

69 At 309 .

70 John Armour, Henry Hansmann and Reinier Kraakman "Agency Problems and Legal Strategies" in Reiner Kraakman and others The Anatomy of Corporate Law: A Comparative and Functional Approach (3rd ed, Oxford University Press, Oxford, 2017) 29 at 30.

71 At 31 .

72 At 30 .

73 Peter Coy and Olga Kharif "This is Your Company on Blockchain" Bloomberg Businessweek (online ed, New York, 25 August 2016).

74 See for example, s 128(3) of New Zealand's Companies Act.

75 Diallo and others, above n 37, at 167. 
principal-agent relationship between shareholders and managers - creates a number of agency costs. ${ }^{76}$ For efficiency reasons, company shareholders delegate business decisions to the board. Shareholders therefore bear the risk that, due to misaligned interests, managers will make unfavourable decisions. These may be mitigated by the creation of supervisory boards, the mandatory inclusion of independent directors, incentivising remuneration schemes, bonding mechanisms and other methods to align manager and shareholder interests. Each of these comes at a significant managerial agency cost.

Rather than decisions being made by a board of directors, governance rules set out in code typically decentralise the decision-making power across DAO token holders. As these token holders are the owners of the $\mathrm{DAO},{ }^{77}$ the division between capital and labour is reduced, and there is, prima facie, no agency cost between ownership and control. ${ }^{78}$ DAO proponents argue that DAO token holders do not face the same agency relationship that company shareholders face through delegated decision-making. ${ }^{79}$ Instead, token holders contribute to the DAO in a non-hierarchical, "dynamic set of working relationships that continuously and dynamically self-organize around projects and outcomes" ${ }^{80}$ Notwithstanding this non-hierarchical structure, as outlined below, although managerial agency problems in the traditional sense may be diminished, new agency issues and similar conflicts may arise instead.

(a) Managerial agency problem

As alluded to above, there is an inherent conflict between a firm's shareholders (principals) and its managers (agents). ${ }^{81}$ When managers are delegated decision-making responsibilities regarding the operation of the firm, a combination of both delegated power and asymmetric information enables managers to act opportunistically and in a manner inconsistent with the shareholders' interests.

Historically, corporate governance strategies have employed control mechanisms to mitigate these conflicting interests. Many of these mechanisms result in agency costs. For instance, monitoring costs arise when costly audits are conducted, managers are fired and new ones hired and when periodic reporting is required.$^{82}$ Similarly, bonding costs arise when managers are subjected to schemes which involve compensation to shareholders if the former fail to act in the latter's best interests.

76 Jensen and Meckling, above n 68, at 309.

77 Alexandra Sims "Blockchain and Decentralised Autonomous Organisations (DAOs): The Evolution of Companies?" (2019) 28 NZ L Rev 423.

78 Voshmgir, above n 19, at 1.

79 Kaal, above n 14, at 19.

80 At 19

81 At 30 .

82 At 19. 
Many of the strategies implemented as a way to align interests between shareholders and managers lack effectiveness in reality. ${ }^{83}$ For example, the mandated inclusion of independent directors on company boards may be futile in the face of CEOs who dominate board discussions. ${ }^{84}$ Ultimately, existing governance mechanisms are effective in some firms and ineffective in others, ${ }^{85}$ and current frameworks are inadequate to address managerial agency problems across a broad spectrum of firms. ${ }^{86}$

At first glance, the managerial agency costs faced by a company are not incurred by a DAO. There need not be incentive mechanisms for decision-makers to act in the owner's interest because those decision-makers are the owners (ie DAO token holders). Firstly, the use of smart contracts eliminates any prospects for individual opportunistic behaviour. De Filippi and Wright propose: "the distributed and disintermediated nature of the underlying blockchain network further ensures - with a high degree of probability - that all codified clauses will perform as planned". ${ }^{87}$ The difficulty in altering smart contracts once they are validated in the blockchain decreases the potential for self-dealing or opportunistic behaviour by modifying the smart contract code. ${ }^{88}$ Secondly, in theory, DAO token holders are solely incentivised to perform work that will increase the value of their token. In essence, this means that it is against their interests to act opportunistically given that this could potentially undermine the value of their tokens.

However, the crypto-anarchist view of managerial agency may be overly straightforward. More recent literature has highlighted potential benefits of managerial agency. ${ }^{89}$ As Pacces suggests, there are positive benefits experienced by corporate controllers, such as managers and majority shareholders, which may reduce the share of the surplus to their principals, but nevertheless create value for the firm that would otherwise be non-existent. ${ }^{90}$ These benefits are "idiosyncratic control rents that are needed to motivate the entrepreneur to undertake firm-specific investments for the firm's

83 James D Cox and Randall S Thomas "Curbing Managerial Agency Costs: Private Litigation and Its Substitutes in the US" in Robin Hui Huang and Nicholas Calcina Howson (eds) Enforcement of Corporate and Securities Law: China and the World (Cambridge University Press, Cambridge (UK), 2017) 221 at 222.

84 Kaal, above n 14, at 9 .

85 For example, differing degrees of the separation of ownership and control do not proportionately reflect the private benefits of control in comparative analyses: see Alessio M Pacces Rethinking Corporate Governance: The law and economics of control powers (Routledge, Abingdon (UK), 2012) at 90.

86 Kaal, above n 14, at 13.

87 De Filippi and Wright, above n 16, at 80.

88 At 81 .

89 Pacces, above $\mathrm{n} 85$, at 89.

90 At $90-91$. 
success". ${ }^{91}$ For example, non-pecuniary benefits, such as that of pride or psychic satisfaction, prove to be important for motivating entrepreneurship. ${ }^{92}$ Pacces argues that these idiosyncratic control rents last beyond a corporation's initial stages of development. ${ }^{93}$ Distributed structures of ownership and control forego these benefits, which cannot therefore be encapsulated in the value of DAO tokens.

Further, although managerial agency undoubtedly results in a number of costs to the firm, it is too simplistic to claim that DAOs eliminate all managerial agency costs. At the heart of a managerial agency problem is the split between ownership and control. This may be removed in the case of a DAO where all token holders are involved in decision-making processes, thereby maintaining control. In the case of the DAO, DAO token holders had a proportionate vote over fundamental investment decisions. ${ }^{94}$ Nevertheless, a novel agency cost arises when considering DAOs further along the autonomous spectrum. With fully autonomous DAOs, decision-making is entirely left to underlying machine learning technology. Although the smart contract's algorithm is coded by humans, who presumably have intentions to be partial owners of the DAO, there is potential for the DAO to selfmanage in a way that fulfils the underlying algorithm but acts against token holder interests.

For example, terra0, a highly autonomous DAO framework created by Paul Kolling, Paul Seidler and Max Hampshire, involves the development of an "augmented forest", where drones and satellites monitor its growth, determining how much wood can be produced and sold. ${ }^{95}$ Although terra0's aim is to generate enough income to pay back its "initiators" (venture capital investors, developers and/or owners of the DAO), buy itself and continue to operate autonomously, in the interim there is potential for the DAO to operate outside of the initiators' interests. For instance, one could hypothesise a scenario where the DAO chooses to sell its timber for a price undesirable to initiators. An autonomous DAO would not act opportunistically per se, as it is bound to abide by the code underlying it. However, with machine learning technology, there is potential for a DAO to make a decision that was never foreseen at the time of coding. It is arguable that, while the traditional managerial agency relationship is eliminated, the delegation of business decision-making to artificial intelligence merely creates a new agency relationship.

This argument of course relies heavily on both a DAO's smart contract incorporating highly advanced machine learning algorithms and on the premise that artificial intelligence can be considered independent from its principal. Such discussion can quickly divulge into debate around attributing legal personhood to artificial intelligence, which is itself a topic worth its own analysis and not within

91 At 93 .

92 At 94 .

93 At 95. But see also Colin Mayer "Firm Control" in Joachim Schwalbach (ed) Corporate Governance: Essays in Honor of Horst Albach (Springer, Berlin, 2001) 69.

94 "Guide to the US Federal regulatory landscape" (16 July 2018) Zephyrnet <https://zephyrnet.com>.

95 Martina Raponi "Terra0, the Augmented Self-Owned Forest" Digicult <http://digicult.it>. 
the scope of this article. ${ }^{96}$ Nevertheless, blockchain enthusiasts should be wary of assuming that DAOs will solve all managerial agency issues.

(b) Decentralising decisions

Proponents of a decentralised society point to a company's hierarchical structure as a major weakness, due to the board amounting to a single point of failure. ${ }^{97}$ It is argued that fraud, incompetence or mere misjudgement by a board of directors will have far-reaching consequences, spreading down the hierarchy. ${ }^{98}$ In contrast, distributing decision-making powers throughout the community of DAO token holders means that there is no single point of failure, so if one member cannot perform, the DAO will continue to operate.

A tangential issue caused by a hierarchical structure is the inefficient use of company member intelligence. Simply, when decisions are solely made by a board of directors, the expertise, knowledge and experience of other employees is not utilised, meaning the company is not making use of the full decision-making power of its members. In contrast, DAOs have the propensity to engage all token holders who propose ideas and areas for improvement.

Decentralising decisions undoubtedly creates a more robust organisation. But how does it impact business efficiency? On a purely theoretical basis, traditionalists will insist that the delegation of decision-making to a board of directors overcomes coordination costs between principals, and thereby promotes efficiency. ${ }^{99}$ For instance, shareholders, especially those of large, listed companies, may face sizeable coordination costs which practically inhibit shareholder decision-making, except for significant decisions. By reverting back to a distributed decision-making process among token holders, DAOs widen the potential for coordination issues.

The magnitude of this problem will vary significantly from organisation to organisation, determined partly by size and partly by engineering. With smaller organisations, reduced coordination costs resulting from fewer shareholders or token holders make distributed decision-making workable. Saying this, a fundamental feature of a DAO is the involvement of many users in the network, spread across a number of different jurisdictions. As it is in the DAO's interests that there are as many users

96 Samir Chopra and Laurence Fredric White "Artificial Agents - Personhood in Law and Philosophy" (paper presented to the 16th European Conference on Artificial Intelligence, Valencia (Spain), 22-27 August 2004).

97 Kelli A Alces "Beyond the Board of Directors" (2011) 46 Wake Forest L Rev 783 at 784.

98 At 784

99 Benjamin E Hermalin and Michael S Weisbach "The Effects of Board Composition and Direct Incentives on Firm Performance" (1991) 20 Financial Management 101 at 103. 
in the organisation as possible, it is unlikely that coordination costs will be mitigated by small numbers. ${ }^{100}$

Nevertheless, many blockchain enthusiasts emphasise the fact that DAO token holders only partake in the organisation because they are there to contribute to the community, such that coordinating token holder engagement will not be an issue. In this way, DAOs resemble cooperative organisations (cooperatives) where "the purpose of the business is to undertake economic activities in the interest of its worker-members, rather than to make a profit for the cooperative itself or external investors". ${ }^{101}$ Whereas cooperatives tend to be localised and carry "significant burden for administration and governance", DAOs naturally involve members from around the globe. ${ }^{102}$ Cognisant of coordination issues, DAOs use incentive mechanisms within their governance structure to encourage member involvement. For example, Horizen, a blockchain platform which provides its own exchange, cryptocurrency and DAO, has implemented an incentive scheme that rewards voters for participating in decision-making. ${ }^{103}$ A society of decentralised organisations with active member involvement may be the dream of many crypto-anarchists, but the practical reality is that financial incentives will lead to the passive investment in successful DAOs through token ownership. As discussed below, voter apathy will undoubtedly be an issue for engineers to grapple with when writing a DAO's underlying code, and this apathy may lead to coordination costs for many businesses.

On top of the risk of organisational inefficiency, blockchain protocols may cause delays due to inherently cumbersome validation processes. More generally, DAOs are subject to the technological and economic restrictions of the underlying blockchain they are founded upon. For example, as Ethereum inherently involves the expensive task of having every Ethereum node execute smart contract codes per the "Ethereum virtual machine", there are currently limitations on the potential complexity of Ethereum based DAOs. ${ }^{104}$ Moreover, the value of a DAO will often be determined by the value of the intrinsic token (cryptocurrency) of the blockchain it is built on. For instance, after its initial coin offering (ICO) The DAO's USD 150 million worth of Ether quickly grew to over USD 250 million as the cryptocurrency (Ether) grew in value. ${ }^{105}$ Of course, a DAO's underlying cryptocurrency may appreciate in value to its benefit, or it may depreciate to its detriment.

100 DAOs are characterised by a network effect, where they become more valuable as more people get involved.

101 Morshed Mannan "Fostering Worker Cooperatives with Blockchain Technology: Lessons from the Colony Project" (2018) 11 Erasmus Law Review 190 at 192.

102 Qayyum Rajan "Ethereum \& the Tao of the Dao" (14 January 2018) Hackernoon <https://medium.com/ hackernoon/>.

103 Horizen Academy "DAO - Decentralized Autonomous Organization" (2019) <https://academy.horizen.global/ horizen/expert>.

104 Alyssa Hertig "Ethereum 101" (13 July 2020) CoinDesk <www.coindesk.com>.

105 Matthew Leising "The Either Thief" (13 June 2017) Bloomberg <https://bloomberg.com>. 
Notwithstanding these external risks and limitations, most decentralised applications are still currently run on the Ethereum network. ${ }^{106}$ Promisingly, blockchain technology is still in its infancy. As these issues are being tackled by developers across the world, inefficiencies present now should not necessarily be an indication of future prospects of DAO complexity.

(c) The issue of the initiators

The absence of a board of directors is often highlighted as a key distinguishing factor of a DAO; it is said to eliminate managerial agency costs and democratise decision-making. However, DAO enthusiasts fail to emphasise that, in reality, DAOs inevitably require a development team to establish their initial framework. Although it is not essential for the development of a DAO-for instance, Tatiana Zalan talks of DAOs which are "born global" due to the globally distributed nature of the blockchain - the collective action required to instigate the development of a DAO in a decentralised manner is extremely difficult in practice. ${ }^{107}$ It is simply not efficacious to use a consensus voting mechanism for the myriad decisions made in a DAO's development phase. ${ }^{108}$

Rather, it is far more efficient for a centralised development team to decide on all preliminary decisions in the build up to producing a "minimum viable product". ${ }^{109}$ Not only is decision-making more efficient, but it is natural for these initiators to want to retain control in these first stages such that they can best achieve their entrepreneurial goals. Voicing this idea in the negative, Jensen and Meckling argue that a manager's incentive to "devote significant effort to ... searching out new profitable ventures" decreases as that manager's ownership claim decreases. ${ }^{110}$ To this date all DAO frameworks have followed this model, including multi-purpose DAO platforms created by Aragon or DAOStack; single purpose DAOs such as The DAO or even arguably the world's most successful DAO, Bitcoin. All of these DAOs were established by a centralised development team or person, including Bitcoin's Satoshi Nakamoto.

The unavoidable consequence is of course that start-up development teams are conferred a considerable amount of power. By making all the fundamental decisions, including those around governance structure and voting mechanisms, the initiators have exclusive control over how they wish to determine the direction, scope and substance of the DAO. Thus, when DAO proponents wax lyrical about the distributed power of a DAO, the role of the initiators is often discounted. It is often said

106 As of 31 July 2019, roughly 800 monthly active developers ran on Ethereum: see Christine Kim "Ethereum: What the Next 4 Years Look Like" (31 July 2019) CoinDesk <www.coindesk.com>.

107 Tatiana Zalan "Born global on blockchain" (2018) 28 RIBS 19 at 20.

108 Charles Okaformbah "Governance in a Decentralized Autonomous Organization" (20 February 2019) Medium <https://medium.com>.

109 Dobrila Rancic Moogk "Minimum Viable Product and the Importance of Experimentation in Technology Startups" (2012) TIM Review 23 at 24.

110 Jensen and Meckling, above n 68, at 313 
that, by deploying DAOs on a blockchain, DAOs become "trustless". ${ }^{111}$ When elaborated upon, this "trustlessness" refers not to the elimination of all trust, but the replacement of trust in humans with trust in the underlying code. ${ }^{112}$ Inevitably, the underlying code has been written by the development team. Therefore, this purported trust in the underlying code is in fact a trust in the competency and good faith of the development team who wrote it. DAO members must trust that the initiators have coded an error free framework and have established sufficiently decentralised governance mechanisms.

Due to this unique source of control, initiators experience a similar position to a board of directors. Like a board of directors, initiators have the power to set the scope and strategy of the organisation, and therefore they also possess the opportunity to make decisions which may further their own interests, at the expense of others. It may be said that development teams which consciously intend to launch their business as a DAO are likely to be well-intentioned, otherwise they would preserve their centralised decision-making power for themselves. This notion fails to recognise the potential for nefarious initiators to scam DAO token holders through fraudulent ICOs. ${ }^{13}$ Even if initiators are well-intentioned and willing to forego their future profits by relinquishing decision making rights, DAO token holders must still trust that initiators are competent and that any inherent biases are not carried forward in code. For example, just as unconscious biases regarding ethnicity, sexuality and gender may jeopardise decision-making by a board of directors, if these biases are held by the initiators, they may be rooted in the DAO's smart contract code.

DAO proponents will of course argue that these issues are mitigated once a DAO is launched. After launching, decision-making power is distributed among token holders, allowing token holders to vote for changes in smart contract code that further their interests. There is no doubt that decisionmaking power is distributed as a result of the launch. However, initiators are likely to retain a considerable proportion of DAO tokens, due to their vested interest in the success of the DAO. Further to this point, the typical requirement of a supermajority for major governance changes on a blockchain means a DAO's underlying framework is difficult to change. ${ }^{114}$ Empirical studies have shown that individuals typically display a strong status quo bias, due to both economic and psychological factors. ${ }^{115}$ Moreover, game theoretical models suggest that there is an "incumbency advantage", where the slightest "benefit of the doubt" given by voters to incumbents dramatically increases barriers

111 Eliza Mik "Blockchains: A Technology for Decentralized Marketplaces?" in Impact of Technology on International Contract Law: Smart Contracts and Blockchain Technologies (forthcoming) at 1.

112 At 1.

113 For context, 80 per cent of ICOs were scams in 2017: see Ana Alexandre "New Study Says 80 Percent of ICOs Conducted in 2017 Were Scams" (13 July 2018) Cointelegraph <http://cointelegraph.com>.

114 De Filippi and Wright, above n 16, at 36.

115 William Samuelson and Richard Zeckhauser "Status Quo Bias in Decision Making" (1988) 1 Journal of Risk and Uncertainty 7 at 47. 
to change. ${ }^{116}$ Combining this incumbency advantage, status quo bias and the potential for voter apathy (as discussed below), there is a likelihood that the initiators' founding governance structures may endure for significant periods, even when it is against the interests of token holders. These factors explain how Bitcoin has failed to undergo any significant reform since its inception, despite wellknown inefficiencies with its underlying proof of work consensus mechanism. ${ }^{117}$

One method to diminish the power of this initial development group is to make a number of core decisions provisional, with confirmation requiring ratification from all DAO token holders once the DAO is launched. In some ways this mirrors the incremental on-chain governance process on Tezos, where amendments require four steps of voting before finally being confirmed. ${ }^{118}$ A provisional approach may be suitable for a limited type of decisions, for example business decisions around product design and pricing. However, the initiators inescapably must decide upon the appropriate governance system (including rules of voting) to ratify those proposals. Consequently, the very means which the wider DAO community has to alter the initiator's decisions, is designed by the initiators. As such, a considerable amount of trust will inherently be put on the initiators to ensure that governance rules sufficiently decentralise power.

\section{Shareholders and token holders}

As discussed above, delegated decision-making to corporate boards creates an agency problem between shareholders and managers. Although shareholders retain certain decision-making powers, they are far removed from a company's normal operations. ${ }^{119}$ To overcome agency problems, shareholders are granted appointment rights and certain decision rights. Appointment rights relate to the shareholder's power to appoint and remove members of the board. ${ }^{120}$ Decision rights are rights which ensure shareholders retain decision-making power for particularly pertinent decisions, for instance the decision to dispose of a sizeable company asset. ${ }^{121}$

DAO tokens proffer similar decision-making rights to token holders. Depending on the smart contract's code, DAO token holders will be able to vote on decisions ranging from appointing

116 Scott L Feld and Bernard Grofman "Incumbency Advantage, Voter Loyalty and the Benefit of the Doubt" (1991) 3 Journal of Theoretical Politics 115 at 130.

117 Alex Galea "Bitcoin development: who can change the core protocol?" (31 March 2018) Medium $<$ https://medium.com>.

118 LM Goodman Tezos - a self-amending crypto-ledger (2 September 2014) at [3.1.4].

119 John Armour and others "The Basic Governance Structure: The Interests of Shareholders as a Class" in Reiner Kraakman and others The Anatomy of Corporate Law: A Comparative and Functional Approach (3rd ed, Oxford University Press, Oxford, 2017) 50 at 51.

120 At 51 .

121 For example, in New Zealand, s 129 of the Companies Act requires all "major transactions" to be approved by special resolution or be contingent on approval by special resolution. 
subcontractors to carry out work, to deciding on the remuneration rewarded for completing work. ${ }^{122}$ As mentioned, DAOs follow a model similar to a cooperative, with owners (token holders) participating in management, and collectively sharing the DAO's resources. ${ }^{123}$

However, DAO tokens do more than grant rights. Tokens are designed as an integral part of the incentive scheme used to manage the conduct of token holders. ${ }^{124}$ Just as cryptocurrencies incentivise the accurate mining and updating of the blockchain, DAO tokens act as financial rewards for those who correctly validate transactions. ${ }^{125}$ It is in the interest of all token holders that the value of the DAO tokens increase, and therefore they will, in theory, endeavour to optimise the DAO through successful projects and accurate validations, and refrain from undermining it through fraud and nonperformance. Notwithstanding the unique characteristics of DAO tokens, a number of issues faced by shareholders, such as voter apathy, agency problems and vote buying, will also apply to token holders.

(a) Voter apathy

Issues of voter apathy and rational ignorance have been considered in both corporate and political contexts for over half a century. ${ }^{126}$ Due to the unlikely probability of an individual vote altering an electoral outcome, costs of investing time and effort to make an informed vote outweigh the utility gained in voting. ${ }^{127}$ For listed companies, shareholder apathy, caused by the opportunity cost of doing due diligence, is a principal reason why decisions are delegated to management in the first place. Relatedly, Easterbrook and Fischel explain how a collective action problem inhibits shareholders from effectively aggregating their voting rights to control a corporation. ${ }^{128}$ This has resulted in shareholder activism falling squarely to hedge funds and other institutional investors which aggregate voting rights. Similarly, if alterations to a DAO's governance code require a large quorum of eligible token holders to vote, DAOs face the risk of a lack of voter participation and an absence of voter engagement. This may be induced by the labour required by each voter to consider each proposal, as has been the case with the BitShares exchange which has faced low voter engagement. ${ }^{129}$

122 Voshmgir, above n 19, at 1.

123 Aste, Tasca and Di Matteo, above n 18, at 23.

124 Voshmgir, above n 19, at 1.

125 At 1 .

126 Anthony Downs An Economic Theory of Democracy (Harper and Row, New York, 1957), as cited in Philip Jones and Peter Dawson "Voter Apathy and 'Rational' Ignorance: Perspectives of the UK 2001 General Election" (University of Bath, Bath, 2003) at 1.

127 Jones and Dawson, above n 126, at 1.

128 Frank H Easterbrook and Daniel R Fischel "Voting in Corporate Law" (1983) 26 JLE 395 at 402.

129 Voshmgir, above n 19, at 1. 
Marcel Kahan and Edward Rock outline four options for an investor with no economic interest in voting. ${ }^{130}$ They either: (1) do not vote at all; (2) cast a less informed vote; (3) look to a voter with an economic interest in voting for suggestions; or (4) are influenced by extrinsic factors (for example, to curry favour with a manager). ${ }^{131}$ In an effort to mitigate option (1) and (2), DAOs may look to engineer a voting system around option (3).

To some success, this has been the case for Tezos. The recent on-chain governance amendments at Tezos saw a voter turnout of over 80 per cent, exemplifying the fact that as governance mechanisms become more sophisticated, issues like voter apathy can potentially be surmounted. One governance system adopted by decentralised systems is liquid democracy. ${ }^{132}$ Liquid democracy involves a "dynamic hybrid" between representative democracy and direct democracy. ${ }^{133}$ On any given issue, voters can choose to delegate their vote to an expert, to present their views, or they can vote for themselves. ${ }^{134}$ Unlike a representative democracy, voters can withdraw their delegation at any time, choosing to delegate to someone else or to vote themselves. ${ }^{135}$ This keeps delegates accountable. Tezos involves a system where delegates (so-called "bakers") vote on behalf of participants in the system. Participants need not do the due diligence themselves, but can instead delegate this job to a baker, much like a retail investor delegating investment decisions to a fund manager. ${ }^{136}$

Many DAO enthusiasts consider liquid democracies to be viable solutions to this voter apathy problem. It is hard to argue that giving voters the option to make their vote more informed will not have better outcomes. However, in practice, the application of a liquid democracy may create a number of issues. For one, the concentration of votes into the hands of delegates is a major step away from the decentralised systems which crypto-anarchists strive for. Arguably, a new agency problem arises where DAO token holders (principals) risk being exploited by the delegates (agents) entrusted with their vote. In this way, option (4) outlined by Kahan and Rock may occur, as these delegates may use extrinsic factors to attract delegation. ${ }^{137}$ A DAO enthusiast may point to the liquidity of the vote which ostensibly ensures that delegates are held accountable, and incentivises them from acting opportunistically. But in practice, the same rational ignorance which causes the token holder to

130 Marcel Kahan and Edward Rock "The Hanging Chads of Corporate Voting" (2008) 96 Geo LJ 1227 at 1263.

131 At 1264.

132 Horizen Academy, above n 103.

133 Horizen Academy, above n 103.

134 Horizen Academy, above n 103.

135 Horizen Academy, above n 103.

136 Arthur Breitman "Why Democracy Doesn't Work in Blockchain Governance - Arthur Breitman (Tezos)" (podcast, 31 July 2019) Blockcrunch 〈http://blockcrunch.libsyn.com>.

137 Kahan and Rock, above n 130, at 1264. 
initially delegate their vote will also extend to their decision to withdraw their delegation or not. In other words, a token holder who delegates their vote because they does not fully understand the complexities of the voting matter, will likely also be unable to understand whether their delegate has misused their vote or not (and it is irrational for them to spend the time and money to educate themselves enough to find out).

(b) Agency problem between controlling and non-controlling token holders

In traditional corporate governance, company owners with a controlling interest in the firm (agents) and those owners with non-controlling interests (principals) may hold conflicting interests. This arises whenever a subset of owners can make decisions which implicate all owners. The classic example is the decision-making power of majority shareholders (agents) over minority shareholders (principals). The imbalance of power, in favour of the majority shareholders, is often reflected in the premium incorporated into "controlling blocks" of tradable shares. ${ }^{138}$ Majority shareholders may benefit from "private benefits of control", ${ }^{139}$ which arise from the potential for both pecuniary gain, such as influence over self-dealing financial decisions, and non-pecuniary gain, ${ }^{140}$ such as "enhanced social status". ${ }^{141}$ Conversely, if minority shareholders hold a veto right, they can in theory become agents (with majority shareholders the principals), as their veto right can effectively control the decisions of all shareholders. ${ }^{142}$

Although awarding voting rights in direct proportion to share ownership (for instance one share carries one vote) aligns economic exposure and control, it leaves minority shareholders vulnerable to opportunistic behaviour by the majority. Granting minority shareholders the right to appoint one or more directors is one way of protecting minority shareholders. For example, in Italy, board representation for minority shareholders is mandatory for listed companies. ${ }^{143}$ More common is the regulation of voting rights, such as those pertaining to dual class equity structures, circular

138 Luca Enriques and others "The Basic Governance Structure: Minority Shareholders and Non-Shareholder Constituencies" in Reiner Kraakman and others The Anatomy of Corporate Law: A Comparative and Functional Approach (3rd ed, Oxford University Press, Oxford, 2017) 79 at 79.

139 Lucian Arye Bebchuk "A Rent-Protection Theory of Corporate Ownership and Control" (National Bureau of Economic Research, Working Paper 7203, 1999), as cited in Pacces, above n 85, at 8.

140 Ronald J Gilson "Controlling Shareholders and Corporate Governance: Complicating the Comparative Tazonomy" (2006) 119 Harv L Rev 1641, as cited in Pacces, above n 85, at 9.

141 Zohar Goshen and Assaf Hamdani "Majority Control and Minority Protection" in Jeffrey N Gordon and WolfGeorg Ringe (eds) The Oxford Handbook of Corporate Law and Governance (Oxford University Press, Oxford, 2018) 449 at 449.

142 Armour, Hansmann and Kraakman, above n 70, at 30.

143 Consolidated Law on Financial Intermediation (Legislative Decree No 58 of 24 February 1998), art 147(3). 
shareholdings and pyramidal ownership structures. ${ }^{144}$ Similar to the granting of appointment rights, sometimes decision rights, for instance the right to bring legal proceedings on behalf of the company, will be granted to a majority of minority shareholders. Likewise, some jurisdictions will require exante approval by a majority of the minority for certain transactions, such as those that could be perceived as self-dealing. ${ }^{145}$ Moreover, the equal treatment norm urges controlling shareholders to act in the interests of all shareholders equally, meaning they must consider the interests of minority shareholders. ${ }^{146}$

Historically, empirical studies showed that jurisdictions with regulations allowing for large private benefits of control (for majority shareholders) experience highly concentrated share ownership. ${ }^{147}$ However, civil law jurisdictions which have since manifested low levels of private benefits, introduced strong equal treatment norms or maintained a relatively higher level of independent directors, continue to exhibit concentrated share ownership. ${ }^{148}$ Enriques and others propose that ownership structures (whether dispersed or concentrated) and the level of protection of minority shareholders are mutually enforcing: controlling shareholders block the enactment of laws that curb their private benefits, while dispersed owners of shares have sufficient political power to demand minority shareholder protection. ${ }^{149}$ It is therefore difficult for ownership structures to change once they are established.

The discussion of majority and minority shareholders becomes particularly pertinent when considering the implications of decentralised governance mechanisms which involve majority and minority token holders. It is arguable that, for DAOs employing a "one token one vote" system, minority token holders face the same risk of exploitation as minority shareholders.

In response, Arthur Breitman (co-founder of Tezos, a public blockchain) argues that shareholders and token holders should be distinguished. He argues that while shareholders have a claim over assets in a company (by virtue of their share), a token is a digital asset itself which only has value because people perceive it as valuable. ${ }^{150}$ Majority shareholders are incentivised to take the pecuniary benefit of the company for themselves, at the expense of the minority shareholder. In contrast, a token holder only receives value from the token by virtue of the network effect; the value of a DAO is that there is

144 Junzheng Shen "The Anatomy of Dual Class Share Structures: A Comparative Perspective" (2016) 46 HKLJ 477 at 479 .

145 Enriques and others, above n 138, at 84.

146 At 86 .

147 Rafael La Porta and others "Law and Finance" (1998) 106 Journal of Political Economy 1113.

148 See Pacces, above n 85, at 7; and Enriques and others, above n 138, at 103-104.

149 Enriques and others, above n 138, at 104

150 Breitman, above n 136. 
a large and diverse community of users. ${ }^{151}$ Breitman concludes that gaining a large, controlling stake will be self-defeating for a token holder.

It is disputable as to whether Breitman's distinction will hold true in practice. Breitman's emphasis on the value of a dispersed token ownership structure may be justified at the extreme level. In essence, if a majority token holder held 80 per cent of DAO tokens, the DAO would lose many of the benefits of decentralisation. However, until an ownership stake surpasses a level which impinges on the network effect, there are still advantages to be had by the majority token holder. A majority token holder can have a greater influence over business decisions and ultimately vote in favour of a change which may benefit their position now, at the expense of the DAO itself. For instance, a majority token holder may vote for the distribution of a dividend payment to token holders at the expense of future research and development funding.

Currently, DAO frameworks have not addressed the potential for majority token holders acting against the interests of the minority. This is not to say that sophisticated engineering could not be utilised to mitigate the potential for opportunistic behaviour occurring. For example, sufficiently large quorums encoded into a DAO's voting mechanisms would ensure majority token holders would require significant coordination to push a motion through. Moreover, the societal norms regarding token holder contribution towards a DAO's goal give rise to similar equal treatment norms found in company law. Notably, the full transparency of a blockchain provides a societal safeguard against majority token holders manipulating a decision to the detriment of the DAO as a whole, as majority token holders will be open to scrutiny. Saying that, this scrutiny is limited in the case of systems where the identity of the token holders is kept anonymous or pseudonymous. To protect the interests of minority token holders, DAOs could look at implementing similar voting ceilings to those imposed by some corporate constitutions, such as limiting a token holder from voting over five per cent, despite having a larger proportion of tokens. Such a rule would be hardwired into the DAO's smart contract code, prohibiting a token holder from skewing a decision by mere force of numbers. Of course, this potentially creates new issues, such as disproportionate levels of control and risk - the underlying reason for a ban on vote ceilings in some jurisdictions. ${ }^{152}$

(c) Vote buying

In recognition that private benefits of control still incentivise DAO token holders to gain a controlling stake of tokens, even if this is limited by a DAO's network effect, vote buying becomes a legitimate issue for DAOs. One imminent means for this occurring is through the aggregation of votes via cryptocurrency exchanges. Many token holders are offered incentives, such as the payment of interest, in return for giving custodian rights to exchanges to hold their tokens. As exchanges

151 Breitman, above n 136.

152 Fernando Vives Ruiz "Iberia: A Little Democracy" (1 July 2010) Law.com <www.law.com/internationaledition>. 
aggregate tokens, they in essence become akin to a financial market's institutional investors, forming the potential to engage in token holder activism.

On a more ad hoc level, token holders with specific interests in getting a proposal voted through could enter smart contracts with individual token holders to purchase voting rights. Like many issues already discussed, token holder activism may be an issue that can be mitigated through sophisticated engineering and the establishment of societal norms. In terms of engineering, smart contracts could encode voting restrictions for tokens that have been traded within a certain temporal proximity to the proposal. For instance, tokens that are traded between the time the proposal is broadcasted to the network and the time voting occurs could lose their voting power. In terms of societal norms, the transparency of token transactions could deter vote buyers from entering into obvious vote buying arrangements for fear of community backlash. Nevertheless, these engineering and societal safeguards are easily circumvented by the availability of off-blockchain deals, which could be made between token holders outside the purview of the DAO community.

\section{Permitted activities, constitutions and governance rules}

Just as the permitted activities of a company were historically set out in a company's constitution, a DAO's purpose and rules will be set out in code, ${ }^{153}$ creating a form of lex cryptographia (private regulatory frameworks based in code). ${ }^{154}$ More specifically, a DAO's purpose and rules of governance are set out in its underlying smart contract(s), and constrained by its underlying blockchain's protocol. ${ }^{155}$ As referred to earlier, the rules of The DAO were outlined in its underlying code, but constrained by the protocol of the Ethereum public blockchain. Governance is therefore two tiered, analogous to a corporation. Firstly, the DAO's smart contract mandates rules similar to a corporate constitution. Secondly, the underlying blockchain protocol limits the DAO's operations similar to mandatory rules outlined in a jurisdiction's corporate legislation.

(a) Complying with constitutional rules

In the case of companies, constitutional rules will normally hold repercussions for those who breach them. Nevertheless empirical examples of corporate collapses illustrate that enforcement of these rules is still an issue. Alexandra Sims argues that there are compliance issues inherent in firms that may be potentially removed by DAOs. ${ }^{156}$ For instance, people may not agree on company rules, even if they do, they may break them, and even when rules are enforced, people and property cannot

153 Aste, Tasca and Di Matteo, above n 18, at 23.

154 De Filippi and Wright, above n 16, at 6.

155 Voshmgir, above n 19, at 1.

156 Sims, above n 77, at 1 . 
easily be restored to their pre-breach state. ${ }^{157}$ In theory, governance rules set out in a smart contract do not bear the same risk of non-compliance. As smart contracts are prescriptive in nature, they are only executed once contractual parameters are fulfilled meaning enforcement issues are negligible. ${ }^{158}$ The parameters and rules of conduct are broadcasted to stakeholders in the blockchain, meaning they are fully transparent. Because the terms of engagement are known to all parties prior to them joining the DAO, involvement in a project is therefore done using opt-in and opt-out mechanisms. ${ }^{159}$

As mentioned, governance rules set out in code technologically limit the capability of people to perform activities beyond what the code allows for. For example, constitutional rules around voting procedure cannot be circumvented as they are hardwired into the code. On its face, this ensures compliance of organisational rules, and in turn promotes a higher standard of legitimacy. Dig a little deeper however and it is apparent that the rigidity of these rules may impinge on organisational efficiency. Sometimes, especially for smaller organisations, there is a practical need to circumvent formal constitutional rules in order to proceed with work. For instance, a company board may be required to reach a 10 person quorum in order to conduct a meeting. Despite notice of a late absentee, the remaining nine directors may choose to proceed with the meeting. In contrast, the autonomous application of a DAO's rigid underlying code would prevent such a meeting from proceeding, perhaps to the detriment of organisational efficiency and commercial expediency.

Moreover, as outlined in Edmund Schuster's critical piece, Cloud Crypto Land, not only does the rigidity of smart contracts fail to accommodate commercial practicalities, it also fails to incorporate legal safeguards fundamental to commercial law. ${ }^{160}$ Notably, commercial parties typically benefit from judicial precedents enabling the avoidance or cancellation of contracts in response to fraud, lack of legal capacity, illegality or contravention of public policy. ${ }^{161}$ As De Filippi and Wright suggest: ${ }^{162}$

Once the wheels of a smart contract are put into motion, the terms embodied in the code will be executed, and they cannot be stopped unless the parties have incorporated logic in the smart contract to halt the program's execution.

Thus this article does not say that flexibility could not be coded into a DAO's underlying smart contract. However, to incorporate plasticity into code is complex, and with every combination and permutation there is another chance for error. Although this should not be a deterrent from

157 At 1 .

158 At 1 .

159 Voshmgir, above n 19, at 1.

160 Edmund Schuster Cloud Crypto Land (London School of Economics and Political Science, LSE Law, Society and Economy Working Papers 17/2019) at 15.

161 At 15

162 De Filippi and Wright, above n 16, at 75 (footnotes omitted). 
development, going forward developers must not only be competent software engineers, but also be cognisant of potential governance and business issues.

(b) Changing constitutional rules

Changing the underlying lex cryptographia of a DAO is very similar to the changing of constitutional rules. Just as a constitution will typically only be changed by a supermajority vote in a general meeting, a change to the underlying smart contract will need to be successfully approved by a majority of token holders.

Even more difficult is the ability to change the blockchain system upon which the smart contracts are built. Similar to the mandatory legislative rules that constrain a company's activities, a DAO is restrained by the consensus protocols for the underlying blockchain for which it is built. However, unlike a company constitution, which will typically have a legislative backstop in the form of statutory default rules when something is not accounted for, ${ }^{163}$ governance rules in a smart contract are intended to be a complete outline of how the DAO should operate. ${ }^{164}$ As such, the engineering of the DAO's underlying smart contract has to be comprehensive before it is launched. If the smart contract code fails to address a particular vulnerability, that vulnerability has the potential to be exploited, without the risk of legislative or judicial intervention.

So-called "on-chain governance" is an experimental field, and involves the very challenging task of changing the blockchain's underlying code. This may involve the majority of all nodes on the network to vote for the desired change, rather than just a majority of DAO token holders. As De Filippi and Wright state: "[t]he technical design of blockchains ... favors the status quo, making blockchainbased networks highly resistant to change." 165

In May 2019, Tezos, a public blockchain that involves a unique proof of stake consensus algorithm, proposed an amendment to its foundational protocol, in a first for on-chain governance. ${ }^{166}$ The amendment, dubbed "Athens", proposed two changes to the blockchain's protocol in order to make transactions more efficient. Unlike other blockchains, like Ethereum or NEO, where the governance of the blockchain is vested in its creators, Tezos was able to instigate an amendment procedure by having all stakeholders (token holders) vote on Athens. The amendment process is a

163 For example, see the New Zealand Companies Act which provides for rules which would otherwise be encapsulated by a company constitution.

164 De Filippi and Wright, above n 16, at 148.

165 At 36

166 Everstake "Tezos on-chain governance in action" (29 May 2019) Medium <https://medium.com>. 
lengthy, four stage process and required a supermajority of votes (that is 80 per cent out of an 80 per cent quorum of token holders) to pass. ${ }^{167}$

Notwithstanding the difficulties of changing something once it is validated on the blockchain, if smart contracts are erroneous (in that they do not allow for what was intended), then the blockchain may have tools at its disposal to correct the problem. An example of this is the hard fork implemented on the Ethereum blockchain after a bug in the code of The DAO was exploited. The DAO, an unincorporated entity, was the first sophisticated DAO of its kind, and operated as a venture capital fund. The DAO's underlying smart contract enabled token holders to vote on projects (proposed by members) to fund, using tangential smart contracts to remit payments as the proposer's milestones were reached. ${ }^{168}$ Any profits from these projects would be redistributed to The DAO's token holders on a pro rata basis. ${ }^{169}$ The DAO's ICO raised over USD 150 million worth of Ether. The DAO's smart contract code had a vulnerability enabling a hacker to siphon off USD 50 million worth of Ether to a clone of The DAO (a "child DAO"). ${ }^{170}$ With roughly 15 per cent of Ether tied up in The DAO, a decision was made to "hard fork" the Ethereum blockchain. ${ }^{171}$ The hard fork essentially involved the rewriting and unwinding of the fraud from the blockchain, resulting in two blockchains: one with the Ether returned to each DAO investor (Ethereum); and one with the hack included (Ethereum Classic). ${ }^{172}$ Controversy around the use of the hard fork continues to this day, with a number of people equating the hard fork to a "too big to fail" sentiment, which may create a bad precedent going forward.

\section{Employment, remuneration and work}

There is increasing emphasis on the duties owed to stakeholders by the firm. ${ }^{173}$ Although the owners of a firm share many interests with external and internal stakeholders, there are various times where these interests diverge. Shareholders or owners (as agents) may act against the interest of stakeholders (principals) by engaging in opportunistic activities, such as "expropriating creditors, exploiting workers, or misleading consumers". ${ }^{174}$ There is significant literature which suggests that it

167 Christine Kim "Welcome to Athens: Tezos Completes 'Historic' First Blockchain Vote" (20 March 2019)

CoinDesk <www.coindesk.com>.

168 De Filippi and Wright, above n 16, at 101

169 At 101 .

170 David Siegel "Understanding The DAO Hack for Journalists" (20 June 2016) Medium <https://medium.com>.

171 Siegel, above n 170 .

172 Antonio Madeira "The Dao, the Hack, the Soft Fork and the Hard Fork" (12 March 2019) CryptoCompare <www.cryptocompare.com>.

173 Business Roundtable Statement on the Purpose of a Corporation (August 2019) at 1.

174 Armour, Hansmann and Kraakman, above n 70, at 30. 
is in the corporation's best interests to undertake labour friendly corporate practices. ${ }^{175}$ However if a firm's investment decision is based solely on shareholder primacy, and a profit-driven criterion, then it will fail to take into account the interests of its other stakeholders, such as employees. ${ }^{176}$ Jurisdictions have attempted to tackle this agency problem by ensuring employees are properly represented. For instance, in Germany, up to half of the supervisory's board members in public companies (Aktiengesellschaft) are employee representatives. ${ }^{177}$ This is reflective of the fact that, left on its own, a corporation will not be incentivised to act in the interests of its employees. ${ }^{178}$

As outlined earlier, a DAO's architecture resembles a cooperative model where token holders receive similar benefits to company shareholders, but also manage and work for the DAO. ${ }^{179}$ Unlike companies that organise work by use of defined roles, standardised employment contracts and ongoing human resource efforts, DAOs self-organise around projects, ${ }^{180}$ embodying what Frederic Laloux labels "[t]eal organizations". ${ }^{181}$ Teal organisations are not unique to DAOs, but DAOs provide an ideal platform for these organisations to be created.

Teal organisations operate on an underlying system of peer relationships where workers retain high autonomy in their domain, and control is spread throughout the organisation, rather than being vested in certain leadership positions. ${ }^{182}$ Similarly, in a DAO, work is conducted through member optimisation proposals, where token holders offer to undertake a project in return for a proposed amount of remuneration. All DAO token holders share the desire to optimise the DAO, and in theory will vote and approve member proposals that will increase the value of their tokens. ${ }^{183}$ Once approval is granted, proposers have the autonomy to take control of their project, with the caveat that they will

175 Olubunmi Faleye and Emery A Trahan "Labor-Friendly Corporate Practices: Is What is Good for Employees Good for Shareholders?" (2011) 101 Journal of Business Ethics 1 at 24.

176 Michael Magill, Martine Quinzii and Jean-Charles Rochet "A Theory of the Stakeholder Corporation" (2015) 83 Econometrica 1685 at 1686.

177 At 1707.

178 At 1708.

179 Aste, Tasca and Di Matteo, above n 18, at 23.

180 Kaal, above n 14, at 19.

181 Coy and Kharif, above n 73, at 10 .

182 Frederic Laloux "The Future of Management is Teal" (6 July 2015) strategy+business <www.strategybusiness.com>.

183 Kaal, above n 14, at 19. 
only be paid once their proposal is completed, per the smart contract. ${ }^{184}$ In this way, DAO token holders who undertake projects are often categorised as subcontractors. ${ }^{185}$

Teal organisations also utilise "agile" practices, which aim to sense and adapt to what the market would like, rather than using "plans, budgets, targets, and incentives". ${ }^{186}$ They are therefore said to have an "[e]volutionary purpose", adapting goals and strategies to meet market demand. ${ }^{187}$ In a similar way, the strategies of DAOs are not set at the top of a hierarchical system, but are shaped by the decentralised needs and wants proposed by DAO token holders in the network.

Where traditional companies often involve employee appraisals and performance reviews to determine promotions and payments, a DAO worker is evaluated solely by its performance. DAO token holders are typically pseudonymous meaning that there are no discriminatory performance measures, and a DAO token holder will be "remunerated regardless of politics, background or education". ${ }^{188}$ DAOs inherently take on a prima facie, non-discriminatory meritocratic ideology, where a member's status in the network is based on reputational capital built by consistent performance, that is, by fulfilling proposals which bring value to the DAO.

The removal of directors (and workers - depending on the DAO's autonomy) ensures that the variable costs incurred day-to-day will be relatively negligible in comparison to that faced by corporations. The use of smart contracts to codify remuneration agreements with subcontractors (DAO token holders proposing to do work for their DAO) also lowers costs due to the elimination of trusted third parties to both monitor and enforce the agreement. ${ }^{189}$ As a DAO's underlying code must both be properly codified to facilitate performance and, to the maximum extent possible, be free from error, the establishment of sophisticated DAOs will incur large setup costs. Notwithstanding this, as software is typically open source, DAOs will be increasingly inexpensive to create.

\section{OBSTACLES}

Many DAO enthusiasts predict a decentralised future: a future beyond legal rules pertaining to particular jurisdictions; a future adopting a paradigm shift in the very idea of economic organisationfrom corporation to DAO. For these crypto-anarchists, lex cryptographia is the only form of law necessary to govern society, and society will follow a predominantly decentralised structure.

184 At 19.

185 Voshmgir, above n 19, at 1.

186 Laloux, above n 182.

187 At 1.

188 Kaal, above n 14, at 20.

189 Chohan, above n 9, at 1. 
This article has endeavoured to insist that these idealistic predictions are not fanciful, but are routinely conveyed in an overly simplistic manner. Not only are there a number of complexities, there are significant obstacles preventing a mainstream uptake of DAOs. Firstly, there has been little discussion by industry experts or academics about the legal status of DAOs. Although blockchain purists may consider this an unnecessary conversation to be had, the reality is that blockchain based organisations will not gain the recognition required to integrate into society until DAOs obtain a feasible legal status. Secondly, the sustainability of a decentralised organisational model has rarely been questioned. The tendency for online services to move towards centralisation will prove to be an obstacle for a mainstream uptake of DAOs. Finally, the power of incumbent institutions to resist the uptake of DAOs will undoubtedly slow progress towards DAO development.

\section{A Legal Indeterminacy}

The purely decentralised society envisaged by many crypto-anarchists does not contemplate specific legal rules. It does not need to. In a perfectly decentralised society, jurisdictional laws and regulations are replaced by lex cryptographia which, by digital architecture, forces people to achieve certain outcomes. ${ }^{190}$ Many legal rules and boundaries can be coded into the rules of smart contracts (for instance data-oriented contracts can cater for many real life scenarios). ${ }^{191}$ Moreover, although complex rules are difficult to encode, artificial intelligence is increasingly enabling the integration of legal rules into code.

However, DAOs do not operate in a vacuum. Regardless of their level of autonomy, DAOs involve human interaction, whether that is through human workers or contractors, human token holders or human customers. Furthermore, if DAOs are to transition to having a recognisable and reputable status in society, they will require legitimacy in the eyes of incumbent institutions to reach that status. Legal determinacy will be an important stepping stone for DAOs to achieve that legitimacy.

\section{Human stakeholders}

\section{(a) DAO workers}

Depending on where a DAO lies on the autonomous spectrum, it will have varying levels of interactions with humans. A DAO with relatively higher human involvement will have human DAO token holders that undertake work for the DAO, and invest capital into the DAO (in exchange for tokens). ${ }^{192}$ It is highly likely that jurisdictions would want to ensure that these DAO token holders

190 Lawrence Lessig "The Law of the Horse: What Cyberlaw Might Teach" (1999) 113 Harv L Rev 501 at 507.

191 Surden, above $\mathrm{n} 27$, at 646.

192 Krysztof Wojdyło and Jacek Czarnecki Blockchain, smart contracts and DAO (Christopher Smith (translator), Wardyński \& Partners, 2016) at 20. 
are treated consistently with other workers in respect to employment law. ${ }^{193}$ For instance, DAO token holders should not face discrimination, unfair dismissal or pay equity issues. The rebuttal is of course that, under a pseudonymous optimisation proposal model, DAO token holders propose their own level of remuneration and amount of work, while circumventing the prospect of appearance based discrimination, so employment law does not apply.

Even so, optimisation proposal models may still need some form of legal rules. By analogy to tender processes used in common law jurisdictions, there could be issues around unfair optimisation proposal processes, such as those that involve biases encoded into the underlying smart contracts. For example, a smart contract's code may arbitrarily prioritise some proposals over others, to the detriment of those who are not seen first by the DAO community. Similarly, there may be opportunities for DAOs to act against the interests of proposers. For instance, some proposal processes (such as that utilised by The DAO) require a token deposit for proposers, so as to prevent an influx of vexatious proposals. ${ }^{194}$ Jurisdictions may want to ensure that DAOs do not exploit potential workers with exorbitant deposits or favour only wealthy token holders who can afford to place deposits. In common law jurisdictions, tender processes are protected by the court's interpretation of a process contract, which gives effect to the reasonable expectations of the parties who engage in it. ${ }^{195}$ In the case of DAOs, proposers will be strictly bound by the architecture of the proposal process encoded into the smart contract. This provides business certainty, but has the potential to entrench any unfavourable terms for potential workers.

(b) DAO consumers

Regardless of employment rights, all DAOs, including those with minimal human involvement, will likely produce goods and services that humans receive. Nation state governments would aim to ensure that these end products meet certain rules and regulations. For example, the New Zealand government would ensure that any DAO selling to New Zealand customers complies with the Consumer Guarantees Act 1993 and Fair Trading Act 1986. To not comply would be to create an unfair playing field between locally regulated corporations and DAOs. Given a DAO's international nature, one could imagine the potential protectionist response by countries who perceive a lack of compliance as a threat to domestic businesses.

193 At 20 .

194 Usha R Rodrigues "Law and the Blockchain" (2019) 104 Iowa L Rev 679 at 701.

195 Peter Devonshire "The Modern Law of Public Tendering: The Principles Defined" (2004) 10 NZBLQ 114, as cited in Jeremy Finn, Stephen Todd and Matthew Barber Burrows, Finn and Todd on the Law of Contract in New Zealand (6th ed, LexisNexis, Wellington, 2018) at [3.2.7]. 


\section{(c) Third party corporations}

Similarly, throughout the course of business DAOs will interact with businesses below and above them in the supply chain, many of which will (at least initially) be legal corporations. For the sake of business certainty, corporations will not enter into contractual arrangements with a DAO unless they know that a potential dispute will be resolved under known law with reliable case history. ${ }^{196}$ For comparison, a major advantage of the corporate form is the fact that company creditors have priority over shareholders, and their personal creditors, to the company's assets. ${ }^{197}$ Asset partitioning allows creditors to refine and streamline monitoring costs over the assets they have claim to. ${ }^{198}$ Knowledge of these legal protections is a major factor in facilitating commerce. In contrast, the uncertainty around stakeholder proprietary rights in DAOs may be too large a risk for many third parties to enter business relations with DAOs at this stage.

(d) Is blockchain transparency a solution to legal uncertainty?

There is a strong argument to be made that the transparency of a blockchain mitigates most of the issues listed above. For instance, the true reason a corporation will be hesitant about contracting with a DAO is not the lack of certainty around the applicable jurisdiction, but the lack of predictability around outcomes. Arguably, there is no lack of predictability because the terms of engagement are clearly outlined in the smart contract, publicly broadcasted on the blockchain. As smart contracts are meant to be comprehensive, the relevant smart contract should outline the remedy for a breach. Crypto-anarchists may go one step further and argue that the immutability and sophisticated engineering of smart contracts will ensure that non-performance and breach of contractual obligations need not be a worry at all for corporations (such as wholesale suppliers or retailers) when considering dealing with a DAO.

This argument, which essentially relies on a solution of full disclosure, can only be taken so far. Grimmelmann emphasises the reliance on human oracles to implement smart contracts. ${ }^{199}$ Oracles are the data feeds necessary to input information into a blockchain that smart contracts require to be executed. As long as oracles are human, data may be erroneously put into a blockchain, so even if information is transparent, it may not be correct. Equally important, while smart contract terms are transparent for all to see, proposers will need to comprehend and digest the terms, outlined in the blockchain's programming language, in full. Just as users of online services generally neglect to read

196 Aurelien Portuese, Orla Gough and Joseph Tanega "The principle of legal certainty as a principle of economic efficiency" (2017) 44 Eur J Law Econ 131 at 137-138.

197 John Armour, Gerard Hertig and Hideki Kanda "Transactions with Creditors" in Reiner Kraakman and others The Anatomy of Corporate Law: A Comparative and Functional Approach (3rd ed, Oxford University Press, Oxford, 2017) 110 at 110.

198 At 110.

199 Grimmelmann, above n 28, at 14 . 
online terms and conditions, everyday contractors and consumers will struggle to read the terms or rules included in smart contracts. ${ }^{200}$ Analogising with modern financial markets, behavioural economics has shown that increased disclosure does not result in tangible beneficial outcomes for consumers. ${ }^{201}$

For corporations, the most effective protection of consumers and suppliers has been through the application of broad, overarching duties and principles, such as duties of care, the duty of loyalty or the principle of good faith. ${ }^{202}$ Broad duties and principles encompass a number of issues which may have been unaccounted for in specific contracts. ${ }^{203}$ It has been argued that the use of principle based regulation creates more business certainty, as a principle is more enduring than narrowly defined rules. ${ }^{204}$ As DAOs currently operate outside of specific jurisdictions, DAO stakeholders fail to benefit from the principle based protections available to company stakeholders. It is unlikely that governments will allow for such an inconsistency to persist in the long run.

\section{Investment in DAOs}

A fundamental element in the establishment process of a DAO is the initial coin offering (ICO). An ICO is a crowdfunding process where DAO token holders transfer funds, in the form of the underlying blockchain's "native" cryptocurrency, ${ }^{205}$ to the DAO, in return for a proportionate allocation of the DAO's token. ${ }^{206}$ Among covering other set up costs, the amount of cryptocurrency raised in the ICO should be enough for the DAO to trigger the necessary smart contracts to operate autonomously. For example, a DAO operating on the Ethereum network requires sufficient Ether to pay for the gas required to execute its operations. ${ }^{207}$

200 Florencia Marotta-Wurgler "Will Increased Disclosure Help? Evaluating the Recommendations of the ALI's 'Principles of the Law of Software Contracts'" (2011) 78 U Chi L Rev 165 at 186.

201 Kendall Grant "From Investor Education to Investor Protection: The Limits of Disclosure and the Way Forward" (2016) 31 BFLR 229 at 237.

202 See for example the New Zealand Companies Act, ss 131-137, which impose broad duties on directors.

203 Julia Black "Regulatory Styles and Supervisory Strategies" in Niamh Moloney, Eilís Ferran and Jennifer Payne (eds) The Oxford Handbook of Financial Regulation (Oxford University Press, Oxford, 2015) 217.

204 John Braithwaite "Rules and Principles: A Theory of Legal Certainty" (2002) 27 ASLP 47 at 48.

205 Stiftung Ethereum "Beginners" Ethereum <https://ethereum.org>.

206 Alex Lielacher "What is a DAICO - A Beginner's Guide" (4 April 2018) Cryptonews <http://cryptonews.com>.

$207 \mathrm{Gas}$ is a unit measuring the computation effort required to execute transactions on the Ethereum blockchain. As the computational effort is costly, those triggering smart contracts must pay miners a proportionate amount of Ether to match the gas required to validate it: see Ameer Rosic "What is Ethereum Gas? [The Most Comprehensive Step-By-Step Guide Ever!]" (2017) Blockgeeks <http://blockgeeks.com>. 
Without sufficient investment, DAOs will be unable to operate. It is therefore imperative that DAO tokens are considered reliable and safe investments. For many blockchain investors, legal indeterminacy of digital assets and even the incidence of ICO scams, has not dampened their popularity. However, if DAOs are to enter mainstream markets such that DAOs can reach the size and scale of incumbent corporations, DAO tokens will have to appeal to everyday investors. For those investors less blasé about the legal status of a DAO token, more certainty and protection will be required.

The failure of The DAO, which resulted in the temporary loss of over USD 50 million worth of Ether, turned a number of heads towards the regulation of ICOs. In 2017 after investigating The DAO hack, the US Securities and Exchange Commission (SEC) released a report ruling that all digital tokens raised through an ICO must comply with United States federal securities laws. ${ }^{208}$ Furthermore any DAO tokens offered and sold in the United States must be registered with the SEC. Notably, the report stated: "The automation of certain functions through this [blockchain] technology, 'smart contracts,' or computer code, does not remove conduct from the purview of the US federal securities laws". 209

Although the report was initially perceived by some as an empty threat, recent enforcement action has shown that the SEC will be willing to impose penalties to those who do not comply. In November 2018, the SEC ordered ICO issuers CarrierEQ Inc and Paragon Coin, Inc to pay USD 250,000 in penalties, register their tokens with the SEC and file periodic reports to the SEC. ${ }^{210}$ Following the SEC's report on The DAO and subsequent statements, ICO issuers are now on notice and should be wary about proceeding without registering with the SEC.

The legal indeterminacy of DAOs themselves, not just DAO tokens, creates an equally large impediment to mainstream investment in DAOs. Whether it is a result of their innovative nature, their ability to operate and be controlled across jurisdictions, or the difficulty of classifying artificial intelligence, a DAO's legal status has so far been undetermined. Without legal recognition, the benefits of limited liability cannot extend to investors. ${ }^{211}$ Instead, DAO token holders may be personally liable, without recourse against the DAO. ${ }^{212}$ For instance, academics have suggested that, by not registering

208 US Securities and Exchange Commission Report of Investigation Pursuant to Section 21(a) of the Securities Exchange Act of 1934: The DAO (Release No 81207, 25 July 2017) at 1.

209 At 2 (footnotes omitted)

210 Kevin Aguirre "Cryptocurrency ICO Issuers on Notice After SEC Seminal DAO Report" (25 January 2019) Fordham Journal of Corporate \& Financial Law <http://news.law.fordham.edu〉.

211 Dean Armstrong, Dan Hyde and Sam Thomas Blockchain and Cryptocurrency: International Legal and Regulatory Challenges (Bloomsbury Professional, Haywards Heath (UK), 2019) at [11.11].

212 At [11.11]. 
as a company, a DAO may be deemed a general partnership. ${ }^{213}$ Associated fiduciary duties between DAO token holders (as partners) may impose obligations unforeseen when choosing to invest or may put a dampener on investment itself. ${ }^{214}$

The SEC's announcements clearly provide the United States position on DAO tokens. Although ICOs have been treated differently in different jurisdictions (for instance China and Pakistan have banned ICOs altogether), the United States position is highly persuasive considering the magnitude and prominence of the market cap of the United States financial markets. ${ }^{215}$ Nevertheless, divergent approaches to determining the legal status of digital tokens may halt investment. Many have hinged the success of blockchain based technologies on the ability of institutional investors to shift capital to digital assets. ${ }^{216}$ Until there is a comprehensive solution to the legal indeterminacy of both DAO tokens and DAOs themselves, they may fail to attract investment from institutional investors.

\section{Jurisdictional complexity}

A further issue faced by a number of online service providers, including DAOs, is an issue of jurisdiction. ${ }^{217}$ An attractive feature of blockchain technology is that nodes are often distributed across the globe. DAOs are therefore potentially subject to any of the rules and regulations of the many jurisdictions that they operate in (or more broadly that they have an influence in). ${ }^{218}$ Lack of harmonisation or unanimity between different jurisdictions creates a large regulatory risk for DAOs. ${ }^{219}$ As mentioned, legal uncertainty can hinder market efficiency. ${ }^{220}$ In essence, the risk of a major jurisdiction, such as the United States, imposing costly regulatory requirements or prohibitive legislation, is a constant factor to be considered and a potential disincentive for investors.

213 At [11.12].

214 At [11.12].

215 Law Library of Congress "Regulation of Cryptocurrency Around the World" (16 August 2019) Library of Congress <www.loc.gov>.

216 Jeffrey Gogo "KPMG: Institutional Investment Key to Cryptoassets Growth" (20 November 2018) Bitcoin News <http://news.bitcoin.com>.

217 Wojdyło and Czarnecki, above n 192, at 21.

218 For example, art 3 of the Regulation 2016/679 on the protection of natural persons with regard to the processing of personal data and on the free movement of such data, and repealing Directive 95/46/EC (General Data Protection Regulation) [2016] OJ L119/1 encompasses any business which targets EU citizens, even if its operations are entirely outside the EU: see art 3(2)(a) and (b).

219 Armstrong, Hyde and Thomas, above n 211, at [11.13].

220 Braithwaite, above n 204, at 48. 


\section{B Tendency towards Centralisation}

The sustainability of a decentralised economy faces significant practical issues. The main argument is that "[n]o matter how decentralized a service is to start with, left to itself, things eventually tend towards centralization". ${ }^{221}$ A popular example of this tendency is the Internet. In the Internet's early days, it was completely decentralised with individual nodes connecting directly. ${ }^{222}$ However with the introduction of Internet service providers (ISPs), the Internet began to become commercialised.

The bundling of services within the Internet value chain has eliminated competition in the market. ${ }^{223}$ For instance, Microsoft's bundling of Internet Explorer and its Windows operating system in 1995 eliminated all other Internet browsers from the market for the time being. More recently, online service providers have monopolised control of traffic, personal data, commerce and the flow of information. Take Alphabet, the parent company of Google, which began as a search engine. Among other things, it has expanded into device and operating systems, with Android and Google phones, internet applications, such as Google Chrome and enabling technologies, such as Google Wallet, DoubleClick advertising and Google Pay. Although the Internet's foundational protocol creates the opportunity for individuals to create web content on an equal playing field, now the large majority of traffic goes through Google or Facebook, giving them a so-called "gatekeeper" status as they control what web content users experience. ${ }^{224}$ As argued by Jonathan Tepper, "the architecture of the internet is still decentralized, the ecosystem of the World Wide Web is not". ${ }^{225}$

Analogising with the Internet, it can be argued that blockchain based services, including DAOs, will become increasingly centralised. To some extent this is already the case. Firstly, many start-up DAOs are created with an inherently centralised structure, by virtue of a localised team of initial developers (see the problem of initiators outlined above). Although they purport to shift towards fully decentralised workforces overtime, how they reach this organisational structure remains to be seen. ${ }^{226}$ It takes a significant entrepreneurial sacrifice to forego this decision-making power. Likewise, there are clear efficiencies with centralised systems which start-up executives may be reluctant to

221 Rahul Matthan "Bitcoin and the law of centralization" (20 December 2017) LiveMint <www.livemint.com>.

222 Vince Tabora "The Evolution of the Internet, From Decentralized to Centralized" (25 March 2018) Hackernoon <https://hackernoon.com>.

223 Mark Page, Christophe Firth and Colin Rand The Internet Value Chain: A study on the economics of the internet (Global System for Mobile Communications Association, May 2016) at 7.

224 Kasper Welbers and Michaël Opgenhaffen "Social media gatekeeping: An analysis of the gatekeeping influence of newspapers' public Facebook pages" (2018) 20 new media \& society 4728 at 4728.

225 Jonathan Tepper "The Death of the Internet" (8 March 2019) The American Conservative <www.theamericanconservative.com>.

226 Wrapious "DAO Brings a Revolution to Working Style" (28 May 2019) <https://wrapious.hk>. 
relinquish. For instance, centralised systems can react to issues promptly, as decisions do not need to be run past an entire network of decision-makers. In this way, centralised systems do not incur the coordination costs of decentralised systems.

Secondly, centralised cryptocurrency exchanges have become a major means for users to store and trade digital tokens. These exchanges operate as third party intermediaries, in some ways defeating the purpose of a decentralised network. Through the use of smart contracts, decentralised cryptocurrency exchanges are possible, but are far from popular with centralised exchanges holding 99 per cent of total cryptocurrency volume. ${ }^{227}$ Moreover, the natural centralisation of miners into mining pools has risked sabotaging the integrity of blockchain systems altogether. For example, in 2014 the mining pool GHash controlled over 50 per cent of the total computational power on the Bitcoin network for over 12 hours, leaving it susceptible to a 51 per cent attack. ${ }^{228}$

Thirdly, centralisation is the preferred means for accumulating both wealth and power. ${ }^{229}$ Naturally, profit-making organisations will tend towards centralised models. Developers will want to retain power and control over their projects. Similarly, majority DAO token holders will like to ensure decisions are made in their interests and that they receive increasing levels of the organisation's profits. ${ }^{230}$ As such, DAOs will likely witness increasingly centralised token holder bases, and moreover, increased power in the hands of controlling token holders.

To take this to the extreme, Schuster suggests that, because properly functioning legal systems "necessitate" hierarchies, any blockchain system wanting to integrate with an existing legal system must become centralised, rendering the core advantage of blockchain technology — decentralisationredundant. $^{231}$

This article does not go as far as Schuster. Undoubtedly, a number of DAOs may be able to persist with their egalitarian and non-hierarchical structures. However, these DAOs must be able to successfully compete with corporations and other centralised organisations to be sustainable in the long run.

227 Karthik Shanmugam "Centralized vs Decentralized Cryptocurrency Exchanges—Explained Simply!" (5 May 2019) Hackernoon <https://hackernoon.com>.

228 Matthan, above n 221, at 1.

229 Grace Rachmany "What Could a DAO Look Like?" (13 August 2019) Hackernoon <https://hackernoon.com>

230 Brady Dale "Everyone's Worst Fears About EOS Are Proving True" (19 September 2019) CoinDesk <www.coindesk.com>.

231 Schuster, above n 160, at 18. 


\section{Incumbent Institutions}

Related to both limitations outlined above is the difficulty DAOs will face as a result of centralised, incumbent institutions. DAO proponents often point to the benefits of decentralising society by circumventing expensive and unnecessary third party intermediaries. The proliferation of DAOs is directly against the interests of many governmental institutions, courts and large corporations in a variety of ways.

DAOs are an immediate threat to governments for various reasons. Their cross-jurisdictional nature makes them difficult to control, and as such they are unlikely to comply with a government's domestic legislation. As such DAOs could provide risks to a nation's citizens, whether it be through financial loss by a failed DAO investment or through an infringement on their human rights. Moreover, DAOs are likely to circumvent government taxes, meaning the proliferation of DAOs will see a reduction in tax revenue. Relatedly, because DAOs operate with cryptocurrencies rather than fiat money they will be unaffected by a government's monetary policy efforts. With a DAO's business operating outside their purview, it is unlikely that governments will allow them to persist without some form of oversight.

Both governments and the courts will be uncomfortable with DAOs operating without a legal status, for the reasons outlined above. As more of a nation's citizens become involved, more pressure will be on governments to integrate a DAO framework into legislation. This will facilitate the development of DAOs in the short-term, but may ultimately restrict the ability for a DAO to expand beyond its statutory limitations.

While regulatory controls imposed by the government may create some restrictions, the crossjurisdictional nature of DAOs may allow them to escape many of these rules, especially if jurisdictions do not harmonise their approaches. Less avoidable is the power of the market. DAOs will have to navigate the responses of powerful multinational corporations, disgruntled by DAO-caused industry disruptions. In this scenario, corporations benefit from being the incumbents. With an existing, loyal customer base incumbent organisations will not have to spend additional funds on building up market share. Moreover, DAOs will have additional costs overcoming the inherent distrust in new unproven innovations, especially those underpinned by artificial intelligence. Similarly, and subject to antitrust laws, incumbent corporations will be able to use anti-competitive practices, such as loss-leading, to drive out competing DAOs.

DAOs do experience advantages which will aid in their competition with corporations. For instance, the ability to draw upon the collective intelligence of their vast member base has potential to aid the organisation in its strategic decision-making. Similarly, their current lack of regulation means that they can avoid expensive regulatory costs that corporations may face. However, one can imagine that large multinational corporations, such as Facebook or Google, which are highly centralised, will feel threatened by DAOs. In fact, Facebook's cryptocurrency Libra exemplifies the ability for incumbent institutions to take ideas from DAOs (for instance a digital financial asset) at 
their expense. A worldwide uptake of Libra might make Bitcoin redundant for the Facebook group's 2.7 billion monthly users. ${ }^{232}$

\section{FUTURE PROSPECTS: A "PARADIGM SHIFT" OR SOMETHING LESS?}

So far this analysis has emphasised the barriers to a decentralised future. However, this article recognises that it is naïve to discount the very real prospects of DAOs in the future. A number of the issues mentioned above point to both the complexity of technology and the stubbornness of attitudes as major limitations to the proliferation of DAOs in the coming years. Attitudes are ever changing and with increasing exposure of the flaws in existing hierarchical structures, DAOs may become the preferred organisational form. Likewise, complex engineering will eventually be able to overcome a number of the practical problems and governance issues DAOs currently face. This will increasingly be the case with the use of artificial intelligence, and by virtue of the large majority of DAOs having open source code.

Nevertheless, there are a number of significant issues that DAOs will need to overcome before they replace all, or even half, of the corporations in the market. As mentioned, legal indeterminacy, a lack of trust from sceptics, backlash from incumbent institutions, alongside myriad governance complications, will prove to be a large barrier to entry.

This article predicts that, in the foreseeable future, we will see a hybrid approach to the integration of DAOs into society. This hybrid approach will be multifaceted. Firstly, there will be a mixture of DAOs and corporations working alongside each other. Secondly, there will be a hybrid regulatory approach to overcome legal status issues. Thirdly, there will be varying degrees of centralisation within DAOs, with some ultimately resembling corporations and some fully autonomous.

\section{A Hybrid Economy: DAOs and Corporations to Coexist}

In many ways the development of E-Commerce has transformed retail markets across the world. ${ }^{233}$ However in 2019, E-Commerce amounted to just 14.1 per cent of global retail sales, indicating that there is still strong demand for offline shopping. ${ }^{234}$ E-Commerce growth in market share has stayed at roughly two per cent consistently over the last five years, and is predicted to

232 John Pavlus "Facebook's Endgame: Getting Inside Your Wallet" (20 September 2019) Fast Company $<$ https://medium.com/fast-company>.

233 Kenneth C Laudon and Carol Guercio Traver E-Commerce 2016: Business, Technology, Society (12th ed, Pearson, London, 2016) at 7.

234 Statista "E-commerce share of total global retail sales from 2015 to 2023" (30 August 2019) <www.statista.com>. 
continue at that rate. ${ }^{235}$ In the meantime, users experience a hybrid between online and offline purchases.

Similarly, DAOs and corporations will co-exist, with DAOs gaining an incremental market share over time. As mentioned above, incumbent corporations have an advantageous position to ensure that they are still relevant and still profitable. These corporations will have no incentive to shift from their existing, centralised structures. However, the development of DAO platforms, which act as DAO facilitating technologies, will assist in the growth of the DAO sector.

For example, the Aragon project, backed by non-profit organisation the Aragon Foundation, ${ }^{236}$ provides open source technology to assist DAO developers. ${ }^{237}$ Aragon delivers a number of different modules for developers to build their own DAOs and decentralised applications. Aragon purports to create its own "digital jurisdiction", ${ }^{238}$ where the functions of DAOs, such as "identity management, ownership, human resources, payroll, voting rights, and even token generation events are all run through the Aragon DAO". ${ }^{239}$ Another example, DAOStack, also provides a modular smart contract framework (along with other services, like user interface templates), so anyone can create a DAO without technical expertise. ${ }^{240}$ DAOStack and Aragon aim to provide a scalable solution for decentralised governance and encourage the creation of DAOs.

With the status quo favouring corporations, but the increasing availability of these DAOfacilitating technologies, the future will witness a hybrid of these two forms of organisation. Those in society who are wary of and have succumbed to the faults of hierarchical institutions will be drawn to DAOs, while less affected persons shall not. For cooperatives, workers in capital intensive sectors, which involve standardised tasks, are relatively indifferent about hierarchical structures compared to workers in knowledge intensive sectors. ${ }^{241}$ By analogy, we may see DAOs become more popular in knowledge intensive sectors, with hierarchical companies persisting in capital intensive sectors.

235 Statista, above n 234.

236 Although Aragon ostensibly aims to become a DAO overtime: see Luis Cuende "Decentralizing Aragon's development II: Minimum Viable Foundation" (2 May 2018) Aragon <https://blog.aragon.org>.

237 Luis Cuende "The Aragon Manifesto" (9 May 2018) Medium <https://medium.com/aragondec>.

238 Luke Duncan "Aragon Network - On a path towards a digital jurisdiction" (25 May 2018) Aragon $<$ https://aragon.org/blog>.

239 Lars Schulze "ICO A DAO - Can Decentralized Autonomous Organizations Save ICOs?" (23 March 2019) Medium <https://medium.com>.

240 Josh Zemel "An Explanation of DAOstack in Fairly Simple Terms" (11 April 2018) Medium $<$ https://medium.com>.

241 Mannan, above n 101, at [2.1]. 


\section{B Interim Legal Solutions: DAOs to Initially Integrate with Legal Systems}

Crypto-anarchists envisage a decentralised society that transcends jurisdictions. For DAO enthusiasts, blockchain protocols and DAO governance mechanisms will be sufficient forms of control. In contrast, incumbent institutions will not want large organisations to operate outside of their control. Governments will want to regulate and ensure they do not forego tax revenue, while corporations will want a level playing field. These competing attitudes will result in a hybrid approach where less powerful DAOs succumb to jurisdictional constraints and larger DAOs attempt to form their own jurisdictions.

The majority of DAOs will need to comply with existing legal structures. To not comply will risk both being stamped out by incumbent institutions and deterring engagement from mainstream market participants (like institutional investors). To overcome these issues, many start-up organisations have adopted a hybrid approach, conforming to recognisable legal statuses but publicly announcing their intentions to shift to a DAO over time. A DAO linked to an entity with the legal capacity to enter into contracts will be able to enter into the business relations required to get off the ground. ${ }^{242}$ At least in the short-term, linking a DAO with a recognisable legal entity allows its initiators to more accurately estimate the potential legal risks of launching it. ${ }^{243}$

An example of a DAO that is ostensibly temporarily linked to a legal organisation is Aragon. As mentioned above, Aragon is organised by the Aragon Foundation, a legally recognised non-profit entity based in Switzerland. ${ }^{244}$ It proposes to "slowly commence the transition of assets to the Aragon Network", which will eventually include transferring all intellectual property to the Network. ${ }^{245}$ Similarly, blockchain platform Horizen is run by the Zen Blockchain Foundation which is a Delaware registered non-profit organisation. ${ }^{246}$ Over time, Horizen aims to transition its foundation to a DAO, starting with decentralising decision-making around the allocation of resources. ${ }^{247}$

Some jurisdictions are opting to meet DAOs halfway, legislating for viable blockchain based corporations. In mid-2018, Vermont passed a bill allowing for the creation of "blockchain-based limited liability companies" (BBLLCs). ${ }^{248}$ In 2019, dOrg, a "blockchain development cooperative",

242 Wojdyło and Czarnecki, above n 192, at 20.

243 At 20

244 Cuende "Decentralizing Aragon's development II: Minimum Viable Foundation", above n 236.

245 Cuende "Decentralizing Aragon's development II: Minimum Viable Foundation", above n 236.

246 Messari "Zen Blockchain Foundation" <https://messari.io>.

247 Horizen Academy "Horizen Expert" (2019) <https://academy.horizen.global>.

248 Stan Higgins "Vermont Governor Signs Bill Clearing Way for Blockchain Companies" (31 May 2018) CoinDesk <www.coindesk.com>. 
became the first BBLLC. ${ }^{249}$ In doing so, it became the first legal entity that directly references blockchain code (open source code from DAOStack) as its source of governance. ${ }^{250}$ In 2018 , Wyoming passed a series of bills which support blockchain innovation, including HB0070 which has the potential to exempt DAO tokens from securities regulation. ${ }^{251}$ While these localised legislative changes undoubtedly encourage blockchain start-ups to base themselves in and boost the local economies, they also provide an avenue for governments to control these new organisations. ${ }^{252}$ Once a DAO is established in a specific jurisdiction, that jurisdiction's governing body has inherently gained regulatory authority over the DAO.

Cognisant of the benefits provided by legal compliance, DAOs are developing new ways to encourage this hybrid approach. OpenLaw, a New York based company, ${ }^{253}$ has developed several open source libraries containing so-called "legal wrappers", for DAOs to incorporate into their code. ${ }^{254}$ OpenLaw has created limited liability wrappers, which extend the corporate veil over DAO business activities, encouraging member involvement by those who would otherwise be deterred due to legal indeterminacy. ${ }^{255}$ Development of these facilitative technologies will help the proliferation of DAOs in the short-term. However, by incorporating, DAOs inhibit future plans to decentralise functions.

Given crypto-anarchists' wariness of regulatory control, it is clear that for many DAOs incorporation will be an interim step towards becoming fully decentralised. Currently these interim frameworks are framed as the only viable option forward, with the alternative that courts force DAOs into undesirable legal frameworks, such as general partnerships which could expose members considerably. However, large online conglomerates have shown that global organisations are not necessarily bound in this way. For instance, Google has outmanoeuvred a number of onerous legal obligations by forum shopping, choosing specific jurisdictions to base the operation of certain services. ${ }^{256}$ Powerful DAOs have potential to take this regulatory arbitrage one step further. Similar

249 Gravel \& Shea "dOrg Launches First Limited Liability DAO" (June 2019) <www.gravelshea.com>; and John Biggs "dOrg Founders Have Created the First Limited Liability DAO" (11 June 2019) CoinDesk $<w w w . c o i n d e s k . c o m>$.

250 Monica Bay "Money" (12 June 2019) <https://law.stanford.edu>.

251 Amin Rafiee "Wyoming: the Blockchain State (Part I)" (20 December 2018) Medium <https://medium.com>.

252 Karl Baker "Delaware eases off early blockchain zeal after concerns over disruption to business" The News Journal (online ed, Delaware, 2 February 2018).

253 Crunchbase "OpenLaw" (2019) <www.crunchbase.com>.

254 OpenLaw "The Era of Legally Compliant DAOs" (27 June 2019) Medium <https://medium.com>.

255 OpenLaw, above n 254.

256 Pamela K Bookman "The Unsung Virtues of Global Forum Shopping" (2016) 92 Notre Dame L Rev 579 at 617. 
to how Facebook's community creates its own rules through social norms and digital architecture, larger sophisticated DAOs have the potential to create their own jurisdictions. For example, Wardunski proposes "a special 'distributed' jurisdiction, different from jurisdictions as traditionally understood". 257 As decentralised systems can operate without many third party intermediaries, by design, DAOs have a greater capability to do this than corporations. However, as the development of an independent online jurisdiction requires a DAO of considerable size and scope, incumbent institutions will be a significant obstacle before we see a DAO capable of achieving this.

\section{DAOs Will Embody Varying Levels of Centralisation and Automation}

Finally, DAOs will be characterised by varying levels of automation and centralisation. As technology improves, processes will continue to be increasingly automated. However, as referred to above, a tendency for online services to trend towards centralisation may restrict the number of organisations that can sustainably maintain a fully decentralised structure.

\section{Automation}

DAOs will vary in levels of automation. Certain business objectives are more predisposed to automation, while others necessarily involve inherently human features. In regard to artificial intelligence, sectors involving creativity, human communication and interpersonal skills will be the last to become fully automated. However, with the integration of various disruptive technologies, such as the Internet of Things, artificial intelligence and 5G, the automated capabilities of DAOs are increasing considerably. Filippi and Wright consider automation as a continuing trend, with "codebased systems are increasingly used to manage the activity of humans and machines". 258 Varying degrees of automation will depend on numerous factors including technological capabilities, applicability of a decentralised governance structure and financial limitations.

\section{Centralisation}

As mentioned earlier, it is almost inevitable that DAOs will begin with a centralised team of developers. The future will see a mixture of blockchain based organisations, some of which will fully transition to decentralised organisations and some of which will decentralise particular functions while maintaining overall control. ${ }^{259}$ Among other factors, the degree of centralisation will be determined by the founders' desire to maintain entrepreneurial direction, the desire for efficiency and

257 Wojdyło and Czarnecki, above n 192, at 21.

258 De Filippi and Wright, above n 16, at 151.

259 Ammous predicts smart contracts to exist "over secured centralized computers operated by trusted third parties with the ability to override them": Saifedean Ammous "Blockchain Technology: What is it Good For?" (2019) 34 BFLR 239 at 247. 
avoidance of coordination costs and how facilitative the legal environment is in the initiators' jurisdiction.

Full decentralisation will require test cases in regard to a number of new, experimental scenarios. For instance, the transitioning from private to public intellectual property ownership remains a legal grey area. Likewise, the durability, efficiency and sustainability of governance mechanisms will have to be tested for organisations to decentralise all their capabilities. If it does become apparent that, with large scale networks, coordination costs are too much, we may see a limit in the expansion of the DAO sector.

In regard to the inherent complexity of DAOs, it is predicted that as more laypeople integrate with blockchain based businesses, technology literacy intermediaries and DAO facilitating technologies, such as Aragon and DAOStack, will gain increasing power. DAO platforms may be a necessary intermediary for non-technical entrepreneurs wanting to create DAOs. Whether these organisations choose to decentralise this newfound power or monopolise it, just as Google profits from its monopoly as a search engine, ironically becomes a question of human nature, and remains to be seen.

\section{CONCLUSION}

Crypto-anarchists and blockchain enthusiasts promulgate the message that DAOs provide innovative, decentralised solutions to century old issues. For those reminded by the grandiose corporate failures of multinational companies, which often occurred at the expense of the innocent individual, these innovative solutions are an attractive alternative option to the corporate form. Unfortunately, these solutions are portrayed in an overly simplistic way, such that a number of complexities are either tactfully dismissed or irresponsibly uncontemplated. The pragmatic reality is that for DAOs to become a viable and competitive business structure, they will need to integrate with existing legal systems. Equally important, DAOs will need to closely monitor their relationships with incumbent institutions, such as competing corporations and wary governments. Moreover, to maintain a decentralised structure, DAOs will have to be consciously structured in a way that not only resists natural tendencies towards centralisation, but also shifts power away from the very developers who set it up. This article does not dismiss these new technological developments as unworkable, but suggests that current academic discourse has not endeavoured to confront these obstacles head on. Ultimately, this article concludes that, notwithstanding improved technology and more sophisticated engineering, we will struggle to reach the decentralised society envisaged by crypto-anarchists, and will instead reach a hybrid society characterised by the coexistence of conventional, centralised, partly decentralised and fully decentralised organisations. 